\title{
A Unified Formulation of Radiative Transfer in Plane-Parallel Atmospheres Based on General Decomposition of Radiance. Part II: An Exemplifying Application to the Hemispherical Harmonics Method with Four Components
}

\author{
BIAO WANG \\ State Key Laboratory of Numerical Modeling for Atmospheric Sciences and Geophysical Fluid Dynamics, Institute of \\ Atmospheric Physics, Chinese Academy of Sciences, and University of Chinese Academy of Sciences, Beijing, China
}

(Manuscript received 24 January 2017, in final form 14 September 2017)

\begin{abstract}
Based on the unified formulation, the so-called hemispherical harmonics method with four components (HSHM4) is derived following the general procedure described in the first paper of this series. The numerical results of this method, including the flux reflections, transmissions, and absorptions for different optical depths, incident beam angles, and single-scattering albedos and the emissions for different optical depths and single-scattering albedos, are reported, in comparison with the results of DISORT with 128 streams as a benchmark. The application of the method to the radiation scheme for climate models is illustrated with the numerical results, including the heating-rate profiles for the shortwave and longwave radiation, radiative fluxes at the top and the bottom boundaries of the atmosphere, and the actinic flux profiles, for typical model atmospheres of Earth and different clear or cloudy conditions. The comparison of results with those of the discrete ordinate methods (DOMs) and the spherical harmonic method (SHM) with four components shows that the HSHM4 has comparable accuracy with the four-stream DOM with double-Gaussian quadrature and is more accurate than the four-stream SHM and the four-stream DOM with full-range Gaussian quadrature in general, especially in the cases of longwave radiation when the multiple-scattering effect should be accounted for. The HSHM4 can be deployed consistently to all of the bands without artificial division between the longwave and shortwave. The development and validation of the HSHM4 exhibit the usefulness of the unified formulation in the study of atmospheric radiative transfer.
\end{abstract}

\section{Introduction}

In Wang (2017, hereafter Part I), a unified formulation for radiative transfer in plane-parallel atmospheres based on the generally decomposed radiative transfer equation system (GD-RTES) has been presented, from which some of the established methods, such as the discrete ordinate method (DOM) and the spherical harmonic method (SHM), can be derived when the appropriate bases are prescribed. Following the general procedure described in Part I, a basis prescribed so that it cannot be transformed linearly from any present one may lead to a new method. However, the applicability of a method to any specific problem, like to Earth's atmospheric problems, should be subjected to close inspection of the numerical results.

The outline of the general procedure to develop a prototype has been presented in Part I. To show more

Corresponding author: Biao Wang,wangbiao@mail.iap.ac.cn clearly how the procedure works, the so-called hemispherical harmonics method with four components (HSHM4) mentioned in Part I is introduced here as a comprehensive example and the numerical results for Earth's atmospheric models are provided, in comparison with those of the DOM and the SHM with four components, which can be developed from the formulation as well.

In the following sections, the parameters of the GDRTES for the HSHM4 are analytically derived (section 2), the solution for homogeneous layers and the numerical results are reported (section 3), and the numerical results for nonhomogeneous atmosphere-surface system are provided (section 4). Also provided are the errors of the results relative to those of DISORT as the benchmark. All of the results are presented along with those of the DOM and the SHM with the same number of components to show the advantages and the disadvantages of the new method. A numerical experiment of modifying the HSHM4 to improve the accuracy in longwave radiation 
problems, also by using the general formulation, is introduced in section 5. Some related issues not explicitly mentioned in sections 2-4 are briefly discussed in section 6 . A summary of the characteristics of the method and its potential application to atmospheric modeling studies is given in section 7. In this paper, when an equation in Part I is referenced, "I." will be prefixed to the equation number.

\section{Basis and GD-RTES of HSHM4}

The HSHM4 is defined with the basis (I.21),

$$
\mathbf{A}=\left[\begin{array}{llll}
H_{0}(\mu) & H_{1}(\mu) & H_{0}(-\mu) & H_{1}(-\mu)
\end{array}\right]
$$

where

$$
\begin{aligned}
& H_{0}(\mu)=\left\{\begin{array}{lll}
1, & \text { when } & \mu \geq 0 \\
0, & \text { when } & \mu<0
\end{array}\right. \\
& H_{1}(\mu)=\left\{\begin{array}{lll}
\mu, & \text { when } & \mu \geq 0 \\
0, & \text { when } & \mu<0
\end{array} .\right.
\end{aligned}
$$

From (I.4) and (I.12), for the HSHM4,

$$
\begin{aligned}
\mathbb{A} & =\left[\begin{array}{cccc}
1 / 2 & 1 / 4 & 0 & 0 \\
1 / 4 & 1 / 6 & 0 & 0 \\
0 & 0 & 1 / 2 & 1 / 4 \\
0 & 0 & 1 / 4 & 1 / 6
\end{array}\right], \\
\mathbb{M} & =\left[\begin{array}{cccc}
1 / 4 & 1 / 6 & 0 & 0 \\
1 / 6 & 1 / 8 & 0 & 0 \\
0 & 0 & -1 / 4 & -1 / 6 \\
0 & 0 & -1 / 6 & -1 / 8
\end{array}\right],
\end{aligned}
$$

and both are invertible. The azimuth-mean scattering phase function is conventionally expanded with the Legendre function into the first four terms as

$$
\begin{aligned}
P\left(\mu, \mu^{\prime}\right)= & g_{0}+3 \mu \mu^{\prime} g_{1}+5 g_{2} P_{2}(\mu) P_{2}\left(\mu^{\prime}\right) \\
& +7 g_{3} P_{3}(\mu) P_{3}\left(\mu^{\prime}\right),
\end{aligned}
$$

where

$$
\begin{aligned}
& P_{2}(\mu)=\frac{1}{2}\left(3 \mu^{2}-1\right), \\
& P_{3}(\mu)=\frac{1}{2}\left(5 \mu^{3}-3 \mu\right),
\end{aligned}
$$

and $g_{0}=1, g_{1-3}$ are the coefficients whose values are determined from the data of the phase function of the atmosphere considered. It can be seen from (I.23), (I.26), and (I.29) that the energy conservation corrections are unnecessary for the HSHM4.

The GD-RTES for the diffuse radiance is (I.8),

$\frac{d \mathbf{I}}{d \tau}=\mathbf{D} \cdot \mathbf{I}-\mathbf{D}_{0} \cdot \frac{\varpi I_{0}}{4 \pi} \exp \left(-\tau / \mu_{0}\right)-\mathbb{E} \cdot(1-\varpi) B(T)$,

where $\mathbf{I}$ is a column vector of the components corresponding to the basis $\mathbf{A}$, that is, $\mathbf{A} \cdot \mathbf{I}=I, \varpi$ is the singlescattering albedo, $\mu_{0}$ is the cosine of the solar zenith angle, $\mu_{0} I_{0}$ is the irradiance of the solar beam, $B(T)$ is the Planck function for temperature $T$, and all boldface letters are formatted as in Part I. From (I.9)-(I.11),

$\mathbf{D}=\left[\begin{array}{cc}\mathbf{D}_{f} & \mathbf{D}_{b} \\ -\mathbf{D}_{b} & -\mathbf{D}_{f}\end{array}\right]$,

$\mathbf{D}_{0}=\left[\begin{array}{c}6-3 g_{1} \mu_{0}-15 g_{2} P_{2}\left(\mu_{0}\right)+\frac{63}{4} g_{3} P_{3}\left(\mu_{0}\right) \\ -6+\frac{45}{2} g_{2} P_{2}\left(\mu_{0}\right)-21 g_{3} P_{3}\left(\mu_{0}\right) \\ -6-3 g_{1} \mu_{0}+15 g_{2} P_{2}\left(\mu_{0}\right)+\frac{63}{4} g_{3} P_{3}\left(\mu_{0}\right) \\ 6-\frac{45}{2} g_{2} P_{2}\left(\mu_{0}\right)-21 g_{3} P_{3}\left(\mu_{0}\right)\end{array}\right]$,

$\mathbb{E}=\left[\begin{array}{c}6 \\ -6 \\ -6 \\ 6\end{array}\right]$,

where

$$
\mathbf{D}_{f}=\left[\begin{array}{cc}
6-3 \varpi-\frac{3}{4} \varpi g_{1}-\frac{63}{64} \varpi g_{3} & 1-\frac{3}{2} \varpi-\frac{1}{2} \varpi g_{1}+\frac{15}{16} \varpi g_{2} \\
-6+3 \varpi+\frac{21}{16} \varpi g_{3} & \frac{3}{2} \varpi-\frac{45}{32} \varpi g_{2}
\end{array}\right],
$$




$$
\mathbf{D}_{b}=\left[\begin{array}{cc}
-3 \varpi+\frac{3}{4} \varpi g_{1}+\frac{63}{64} \varpi g_{3} & -\frac{3}{2} \varpi+\frac{1}{2} \varpi g_{1}+\frac{15}{16} \varpi g_{2} \\
3 \varpi-\frac{21}{16} \varpi g_{3} & \frac{3}{2} \varpi-\frac{45}{32} \varpi g_{2}
\end{array}\right] .
$$

\section{Results for homogeneous layers}

For a homogeneous layer $l$, with the top at the optical depth $\tau_{l-1}$ and the bottom at $\tau_{l}$, the eigenvalue problem of $\mathbf{D}$ is solved first to get the matrices of the eigenvalues $\mathbf{k}$ and of the eigenvectors $\mathbf{G}$. The particular solution $\mathbf{Q}$ can be obtained after (I.43). Then the general solution for the layer can expressed with the operators $\mathbf{S}, \mathbf{S}_{0}$ (including $\mathbf{R}_{0}$ and $\mathbf{T}_{0}$ ), and $\mathbf{B}^{ \pm}$[see (I.65)-(I.67)].

A common practice to show the numerical results for homogeneous layers is to draw the values of the plane reflection, transmission, and absorption to the solar beam as functions of the optical depth $\tau$ and cosine of the solar zenith angle $\mu_{0}$ (e.g., King and Harshvardhan 1986; Liou et al. 1988; Li and Ramaswamy 1996; Zhang and Li 2013), when the thermal emission of the atmosphere can be neglected. From (I.70), (I.105), and (I.106),

$$
\begin{aligned}
& R_{0}=f_{+}\left(\tilde{\mathbf{A}}^{+}\right) \cdot \mathbf{R}_{0} / \mu_{0}, \\
& T_{0}=f_{-}\left(\tilde{\mathbf{A}}^{-}\right) \cdot \mathbf{T}_{0} / \mu_{0} .
\end{aligned}
$$

The total transmission is

$$
T_{0}+\exp \left(-\tau / \mu_{0}\right)
$$

and the absorption is

$$
A=1-R_{0}-T_{0}-\exp \left(-\tau / \mu_{0}\right) .
$$

For the HSHM4, from (I.21) and (I.70),

$$
f_{ \pm}\left(\tilde{\mathbf{A}}^{ \pm}\right)=\left[\begin{array}{ll}
\pi & 2 \pi / 3
\end{array}\right],
$$

where $\tilde{\mathbf{A}}^{ \pm}$has been defined in (I.56) but is identical to A for the HSHM4.

Figure 1 shows the numerical results of the flux reflection, the total flux transmission, and the absorption as functions of optical depth and cosine of the solar zenith angle for four values of the single-scattering albedo $(0.99,0.9,0.8$, and 0.5$)$, calculated with the HSHM4. The Henyey-Greenstein phase function with the asymmetry factor $g=0.84276$ after King and Harshvardhan (1986) is adopted. The $\delta-N$ scaling (Wiscombe 1977) is implicitly applied to all methods mentioned in this paper when the numerical results are evaluated so that the prefix $\delta$ - is omitted everywhere. The figure shows results for 100 values of $\tau$ spaced evenly on a $\log$ scale between 0.1 and 100 and 64 values of $\mu_{0}$ at the half-range Gaussian quadrature abscissas between 0 and 1 . The deviation of the results from those of DISORT (Stamnes et al. 1988) with 128 streams (DST128) is shown in Fig. 2. In terms of absolute error, the HSHM4 is seen to be accurate to within 0.01 in reflection for $\tau \lesssim 1$ and $\mu_{0} \gtrsim 0.2$, or for $\tau \gtrsim 1$ and $\mu_{0} \gtrsim 0.15$, and to be accurate to within 0.01 in transmission for $\tau \gtrsim 1$ and $\mu_{0} \lesssim 0.3$ or all optical depths when $\mu_{0} \gtrsim 0.3$. The absorption calculated by HSHM4 is accurate to within 0.01 for all optical depths when $\mu_{0} \geq 0.2$.

For comparison, an SHM prototype and two DOM prototypes with four components (denoted as SHM4, DOM4a, and DOM4b, respectively) are also derived from the unified formulation. The prescription of the SHM4 has been given by (I.16) and (I.61). The DOM4a and the DOM4b are both after the prescription (I.20); the DOM4a uses the Gaussian quadrature, that is, $\mu_{1}=$ $0.8611363, \mu_{2}=0.3399810$, and $w_{1}=0.3478548$ (Liou et al. 1988), while the DOM4b uses the double-Gaussian quadrature (Thomas and Stamnes 1999), that is, $\mu_{1}=$ $0.7886751, \mu_{2}=0.2113249$, and $w=0.5$. The DOM4b is actually equivalent to DISORT with four streams (DST4), and we find, as a validation of the unified formulation, the two implementations obtain the same results as they are supposed to. Therefore, only one of them will be referred to in the following discussion.

The results of planar reflection, transmission, and absorption are calculated with these methods, too. The deviations of the results from those of the DST128 are provided in Figs. 3-5, respectively. The results of the SHM4 and the DOM4a were reported previously by the independent works of other authors (Liou et al. 1988; Zhang and Li 2013).

It can be seen that the deviation pattern of the SHM4 (Fig. 3) is quite similar to that of the DOM4a (Fig. 4), but the ridge and trough of the former are a little greater than those of the latter. A closer comparison of the results shows that only in very limited areas such as those around $\mu_{0} \approx 0.1$ are the reflection and absorption of the DOM4a and the SHM4 more accurate than those of the HSHM4. The result of the DST4 (Fig. 5) is quite similar to that of HSHM4, but the DST4 can be seen to be a little more accurate around the moderate solar zenith angles $\left(\mu_{0} \approx 0.4\right)$ and optical depths $(\tau \approx 2)$. The theoretical issues related to the similarities of the two pairs of methods will be discussed in section 6 .

The thermal emission of a homogeneous layer calculated by the HSHM4 is compared to that of the other methods, too. The apparent emission at the top of a layer is defined as the ratio between the upward irradiance of 


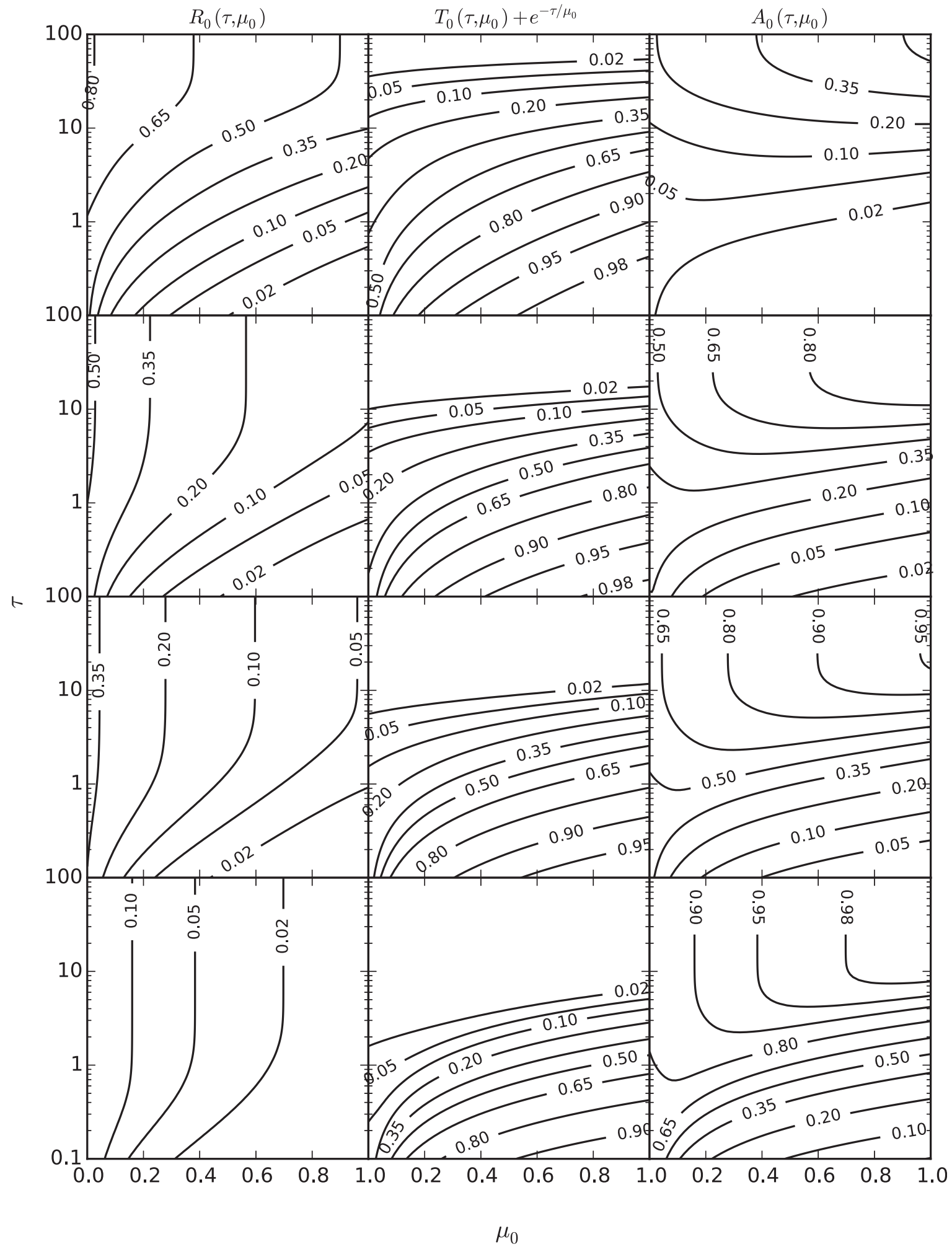

FIG. 1. (left) The flux reflection, (middle) the total flux transmission, and (right) the absorption as a function of optical depth $\tau$ and cosine of the solar zenith angle $\mu_{0}$ for four values of single-scattering albedo [(top to bottom) $0.99,0.9,0.8$, and 0.5 ] of a homogeneous layer, calculated with the HSHM4. 


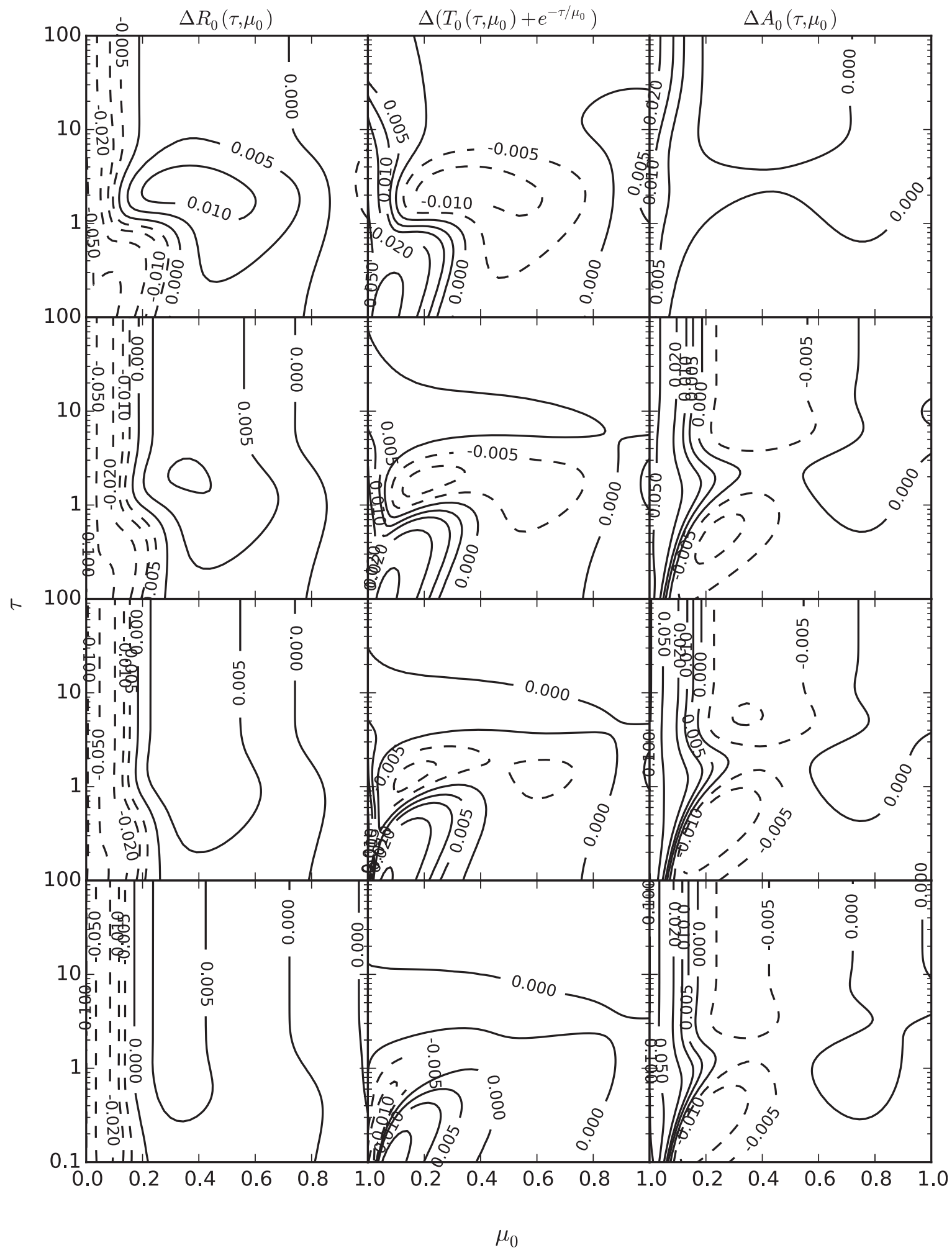

FIG. 2. The deviations of the numerical results of the HSHM4 shown in Fig. 1 from those obtained with the DISORT program with 128 streams. 


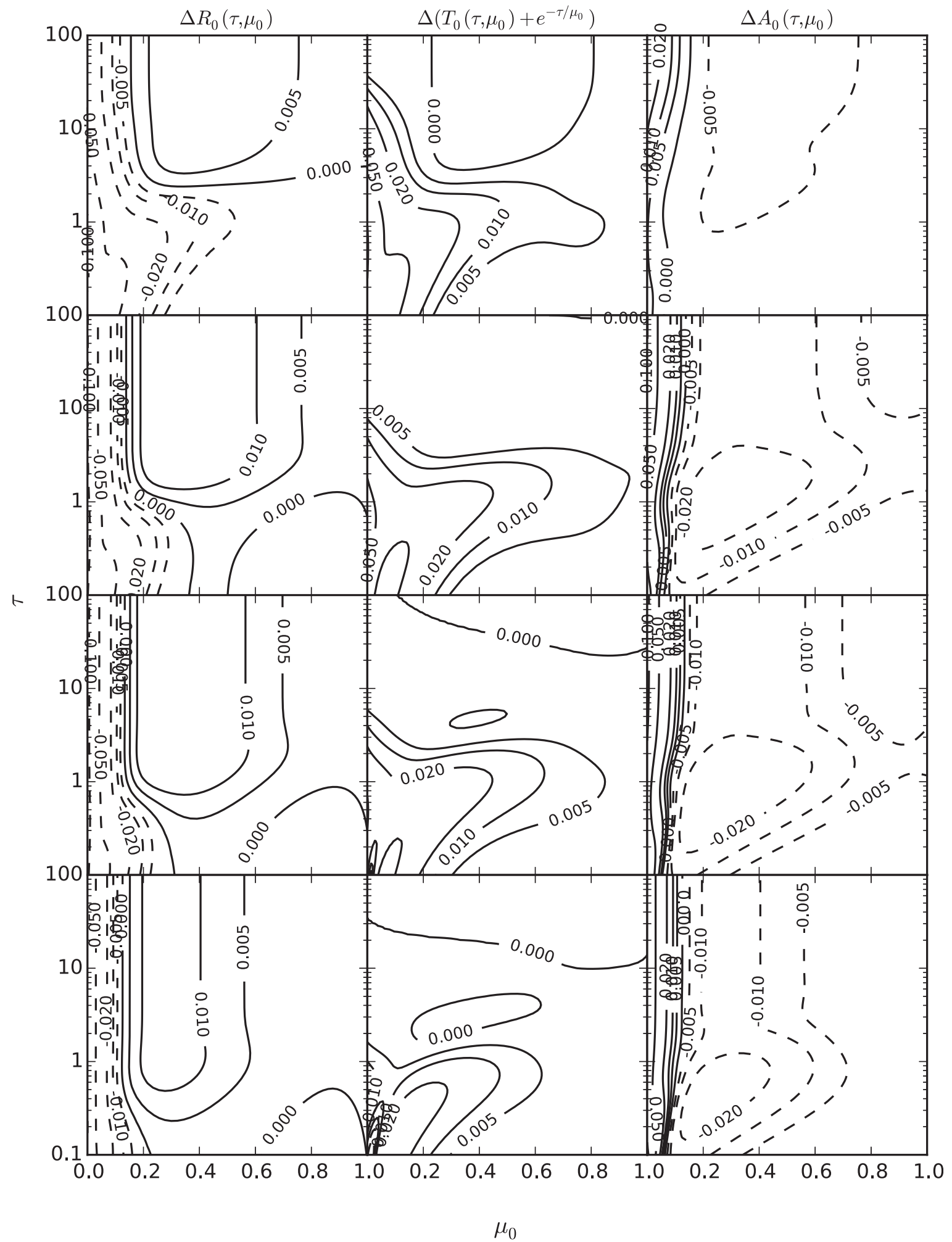

FIG. 3. As in Fig. 2, but for the SHM4. 


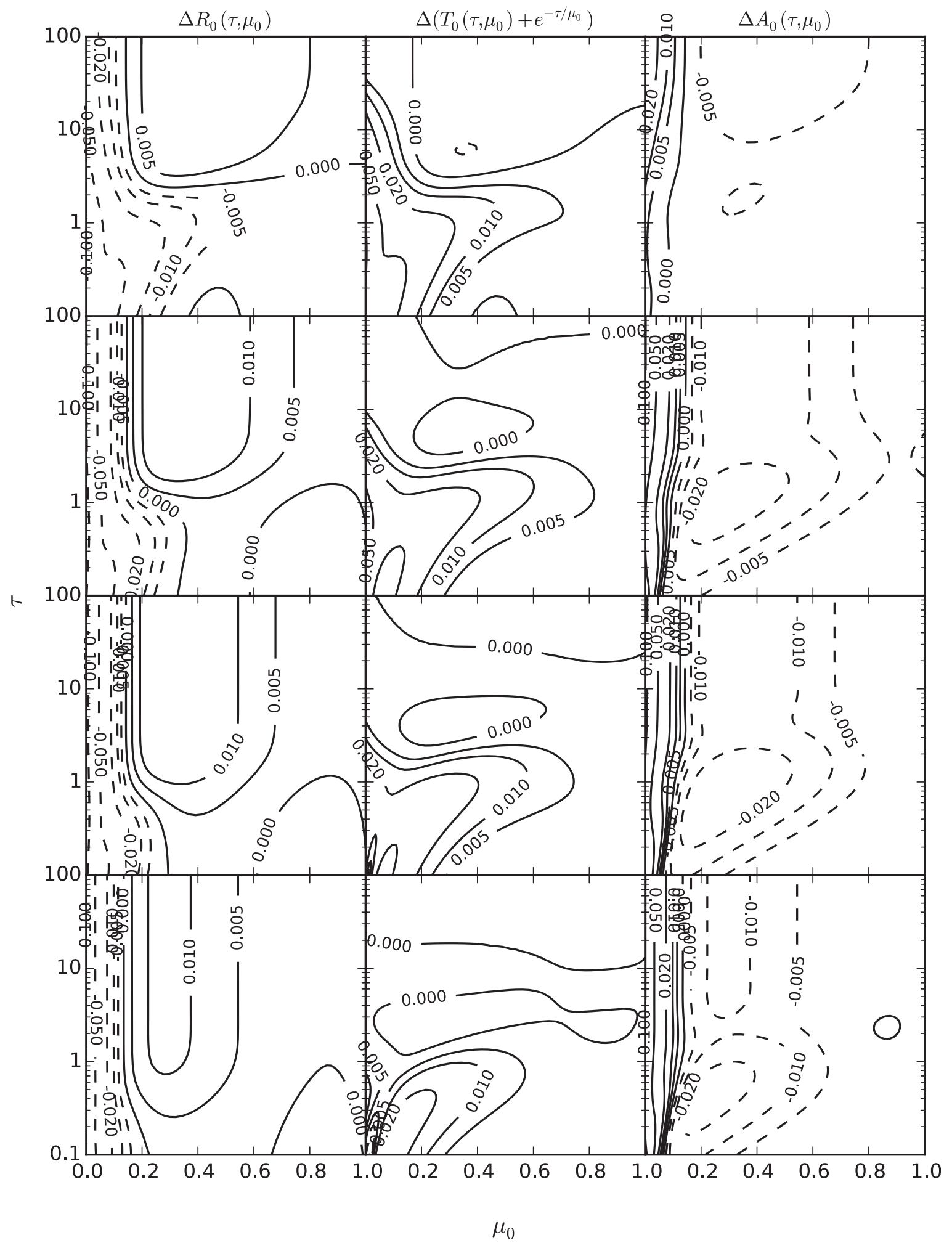

FIG. 4. As in Fig. 2, but for the DOM4a. 


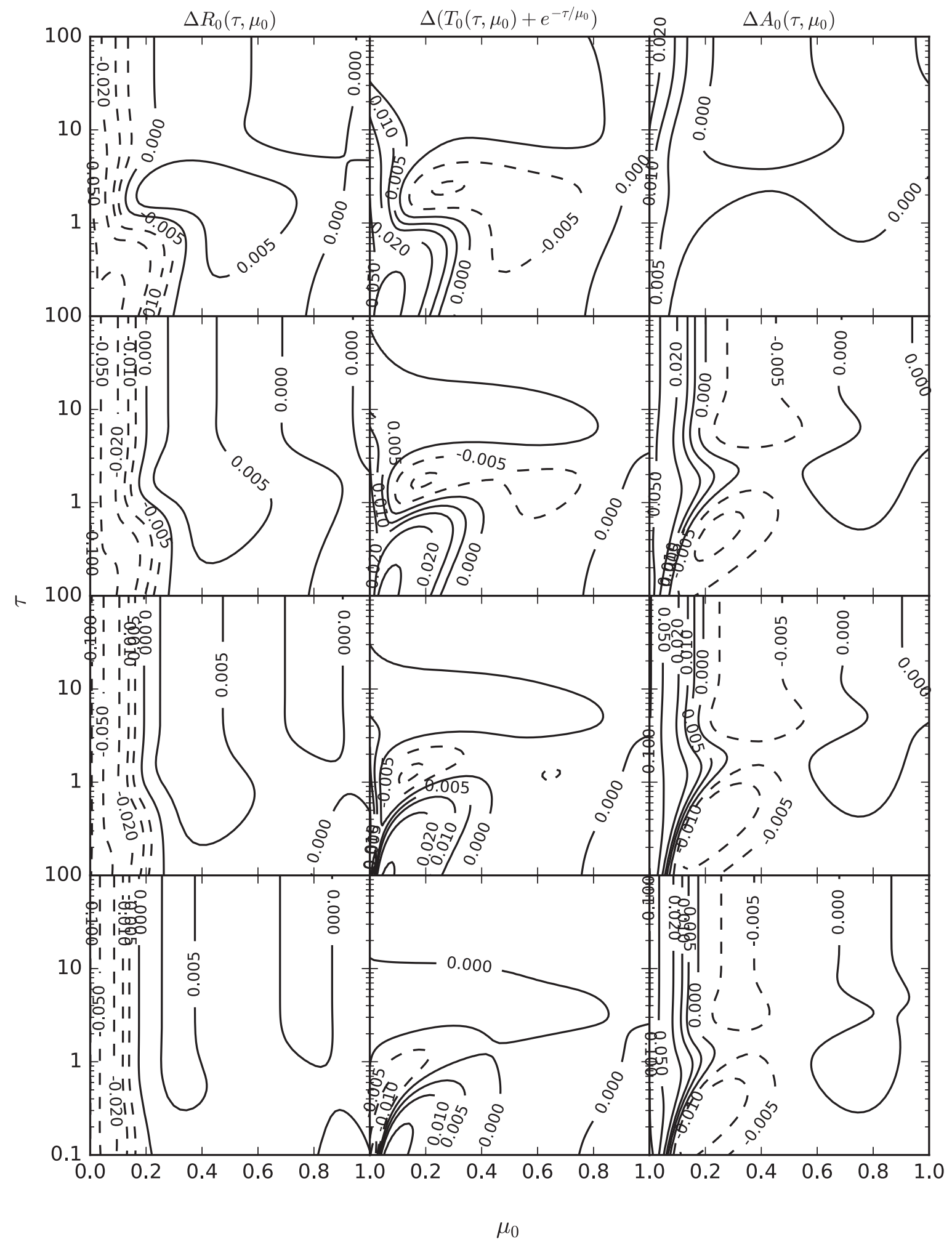

FIG. 5. As in Fig. 2, but for the DST4. 
the layer and that of the blackbody for the temperature of the top, when no external source (solar beam or diffuse radiation incidence) or boundary exists,

$$
\varepsilon^{+}=\frac{B^{+}}{\pi b_{0}},
$$

where $B^{+}$has been defined by (I.108) as

$$
B^{+}=f_{+}\left(\tilde{\mathbf{A}}^{+}\right) \cdot \mathbf{B}^{+} .
$$

With the unified formulation, the scattering process is treated explicitly in the presence of thermal emission.

In Earth's atmosphere, the thermal emission occurs mostly at wavelengths greater than $4 \mu \mathrm{m}$ and the scattering properties are essentially contributed by clouds and aerosols. The single-scattering albedo of water clouds for the longwave radiation can vary from about 0 to 0.9 , and the asymmetry factor can be from about 0 to almost 1 (Hu and Stamnes 1993), while the gases' absorption always dilutes the single-scattering albedo of the layer as a whole. Figure 6 shows the apparent emissions of a homogeneous layer with $g=0.5$ and $\varpi=0.0,0.3,0.6,0.9$, calculated by the DST128, the DST4, the HSHM4, the SHM4, and the DOM4a, as well as the deviations from the DST128. The apparent emission can be seen to increase with the optical depth before reaching a saturated value, which should be unity for $\varpi=0$ and decrease with the single-scattering albedo. The HSHM4 and the DST4 yields significantly better results than the DOM4a and the SHM4. The results of the HSHM4 and the DST4 are very close to each other and indistinguishable in the figure. However, the DOM4a and the SHM4 notably fail to converge to the correct saturated value and the relative error can be as large as $7 \%$, though it seems less serious when the single-scattering albedo is sufficiently large.

The calculations have been made for different asymmetry factors (from 0.1 to 0.9 ) and for layers with temperature gradients $\left(b_{1} \neq 0\right)$, but the conclusion is not really altered, so the results are not shown here.

\section{Results for Earth's atmosphere}

Following the adding operations described in section $3 c(4)$ of Part I, the radiative flux and heating rate are calculated with the four methods (HSHM4, SHM4, and DOM4ab). The absorption of the gases $\mathrm{H}_{2} \mathrm{O}, \mathrm{CO}_{2}, \mathrm{O}_{3}, \mathrm{~N}_{2} \mathrm{O}, \mathrm{CO}$, and $\mathrm{CH}_{4}$ is represented with a $k$-distribution scheme, which has been used in an early version of the climate model FGOALS (Wang et al. 2000). All calculations in this section are made with the same $k$-distribution scheme so that the accuracies of the different radiative transfer methods can be compared. The scattering effects of gases' molecules and cloud droplets are considered. The surface is simply assumed as a Lambertian surface with albedo $R_{s}=0.2$ for solar radiation and 0.0 for terrestrial radiation. For the HSHM4, it can be seen that the renormalization for the decomposed surface reflection and emission (I.83) is unnecessary, that is,

$$
\hat{\mathbb{A}}^{+*}=\hat{\mathbb{A}}^{+}=\left[\begin{array}{l}
1 \\
0
\end{array}\right]
$$

and from (I.77)-(I.81), the operators for the surface are

$$
\begin{aligned}
\mathbf{R}_{s}= & R_{s}\left[\begin{array}{cc}
1 & \frac{2}{3} \\
0 & 0
\end{array}\right], \\
\boldsymbol{\Sigma}_{s}^{+}= & {\left[I_{0} \mu_{0} R_{s} \exp \left(-\tau_{s} / \mu_{0}\right) / \pi\right.} \\
& \left.+\left(1-R_{s}\right) B\left(T_{s}\right)\right]\left[\begin{array}{l}
1 \\
0
\end{array}\right],
\end{aligned}
$$

where $\tau_{s}$ is the total optical depth of the atmosphere above the surface and $T_{s}$ is the surface temperature.

The shortwave flux and heating rate are calculated for the U.S. Standard Atmosphere, 1976 profile (COESA 1976), with three solar zenith angles $\left(\mu_{0}=0.1,0.5,0.9\right)$, and three liquid water path (LWP) conditions (LWP = $\left.0,10,100 \mathrm{~g} \mathrm{~m}^{-2}\right)$. The radiative parameterization of water cloud developed by $\mathrm{Hu}$ and Stamnes (1993) is adopted with the effective radius prescribed as $10 \mu \mathrm{m}$. For the purpose of comparing the different methods, the effect of cloud height is found not significant to the conclusion so that only the results for the moderateheight cloud (with the top and the bottom at 4.2 and $5.6 \mathrm{~km}$, respectively) occupying two model layers are shown to get a fair view of the profiles.

Figure 7 shows the results for the moderate solar zenith angle, $\mu_{0}=0.5$. For the clear-sky condition $(\mathrm{LWP}=0)$, the heating-rate profiles obtained by the four methods have good accuracies (within $0.05 \mathrm{~K} \mathrm{day}^{-1}$ ) and the HSHM4 and the DST4 are obviously better at the altitudes above $10 \mathrm{~km}$. For the thin-cloud condition (LWP = $10 \mathrm{~g} \mathrm{~m}^{-2}$ ) where quite a fraction of solar beam can penetrate the cloud for the moderate solar zenith angle, the heating-rate profile obtained by the DOM4a seems slightly more accurate than those by the HSHM4 and the DST 4 above about $25 \mathrm{~km}$, but the HSHM4 and the DST4 give the better results within and below the cloud. The HSHM4 and the DST4 overestimate the heating rate in the upper layer of cloud and underestimate it in the lower, but in terms of the energy budget of the cloud layers, the results are better than those of the DOM4a and the 

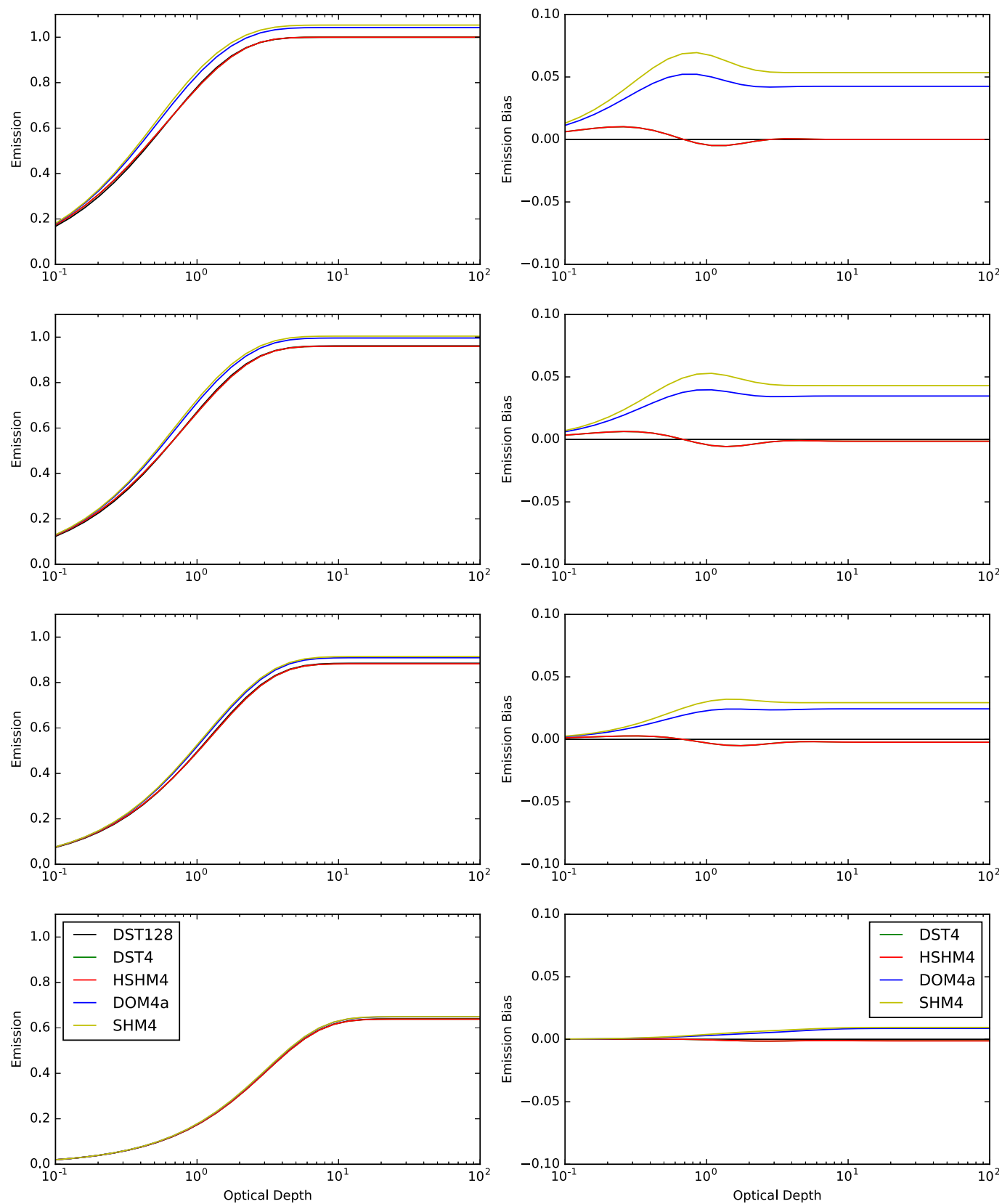

FIG. 6. (left) The apparent emissions of a homogeneous layer as functions of optical depth calculated with the DST128, the DST4, the HSHM4, the DOM4a, and the SHM4 and (right) the deviations from the DST128 results for four values of the single-scattering albedo [(top to bottom) 0.0, 0.3, 0.6, and 0.9].

SHM4, which underestimate the heating rate in both layers. For the thick-cloud situation $\left(\mathrm{LWP}=100 \mathrm{~g} \mathrm{~m}^{-2}\right.$ ) when virtually no direct beam exists below the cloud, the heating-rate profile of the DOM4a seems better than those of the HSHM4 and the DST4 at most altitudes, except for those within and below the cloud, especially when the energy budget of the cloudy layers is considered. In this case, the HSHM4 and the DST4 show slightly different results within the cloud layers. The HSHM4 yields a little lower heating rate in the upper layer, and the DST yields a little higher heating rate in the lower layer. The difference between the HSHM4 and the DST4 at the altitudes above $30 \mathrm{~km}$ should be from the roundoff errors of the different numerical procedures.

Figure 8 shows the results for the low solar elevation $\left(\mu_{0}=0.1\right)$. It can be seen that the results of the HSHM4 

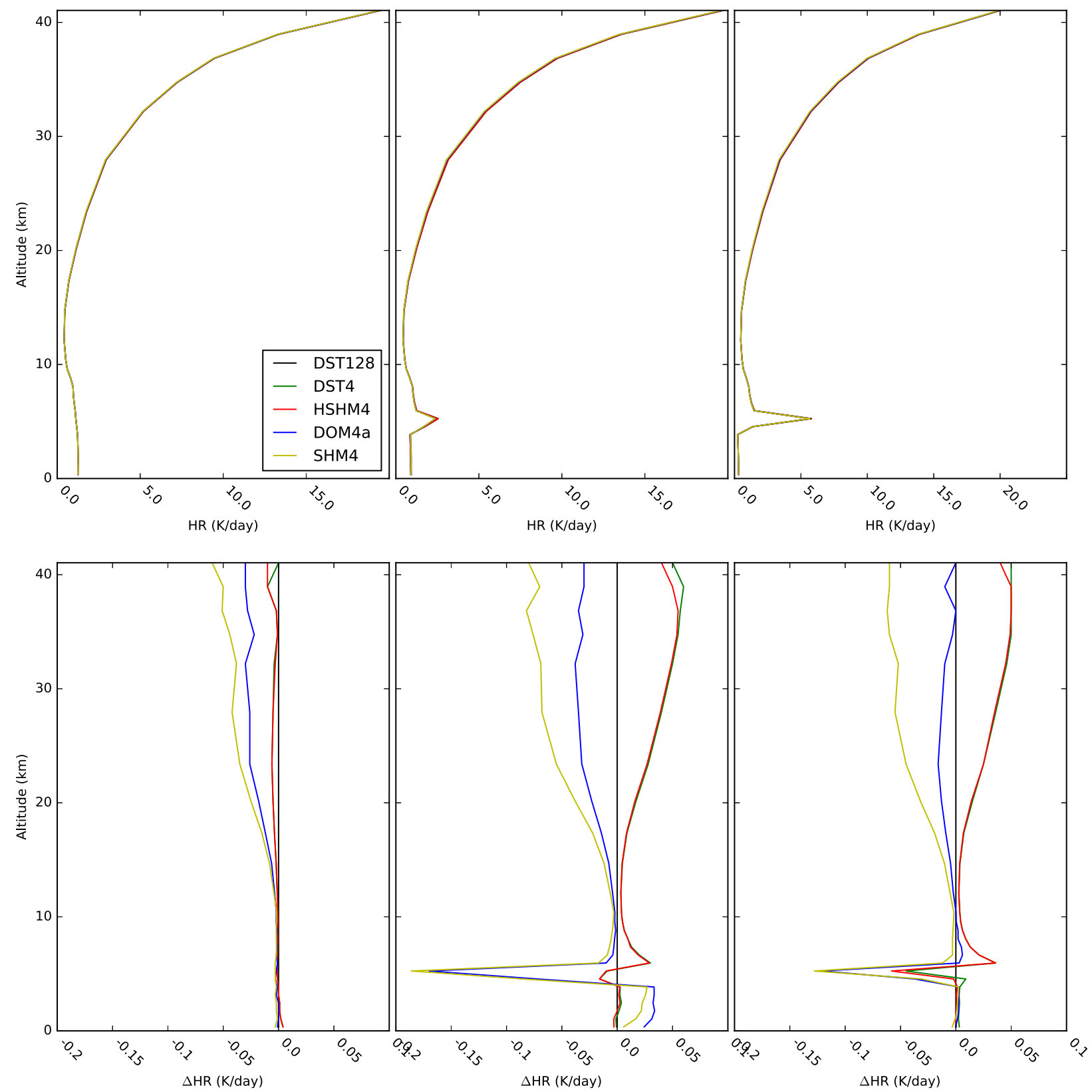

FIG. 7. The profiles of (top) the heating rate for the STD calculated with the DST128, the DST4, the HSHM4, the DOM4a, and the SHM4 for $\mu_{0}=0.5$ and middle-cloud LWP $=$ (left) 0 , (middle) 10 , and (right) $100 \mathrm{~g} \mathrm{~m}^{-2}$ and (bottom) the errors relative to the DST128 results.

and the DST4 are better than the other two methods at almost all altitudes for the three clear or cloudy cases. The results of the HSHM4 and the DST4 are almost indistinguishable for all the three clear or cloudy cases. When the solar elevation is high $\left(\mu_{0}=0.9\right.$; Fig. 9$)$, the heatingrate profile obtained by the HSHM4 and the DST4 is also better than those by the DOM4a and the SHM4 at most altitudes and the heating rates of the DST4 in the thick cloud layers are little better than those of the HSHM4.
Table 1 lists the upward fluxes at the top of the atmosphere $F_{\mathrm{TOA}}^{+}$, the downward fluxes at the surface $F_{\mathrm{Srf}}^{-}$, and the total absorption by the atmosphere, calculated by the DST128 and the relative errors of the corresponding results calculated by the other methods, for each of the nine cases mentioned above. The boldface type indicates where the accuracy is better than $1 \%$. It can be seen that every method shows reasonable accuracy for the shortwave radiation. The results of the 

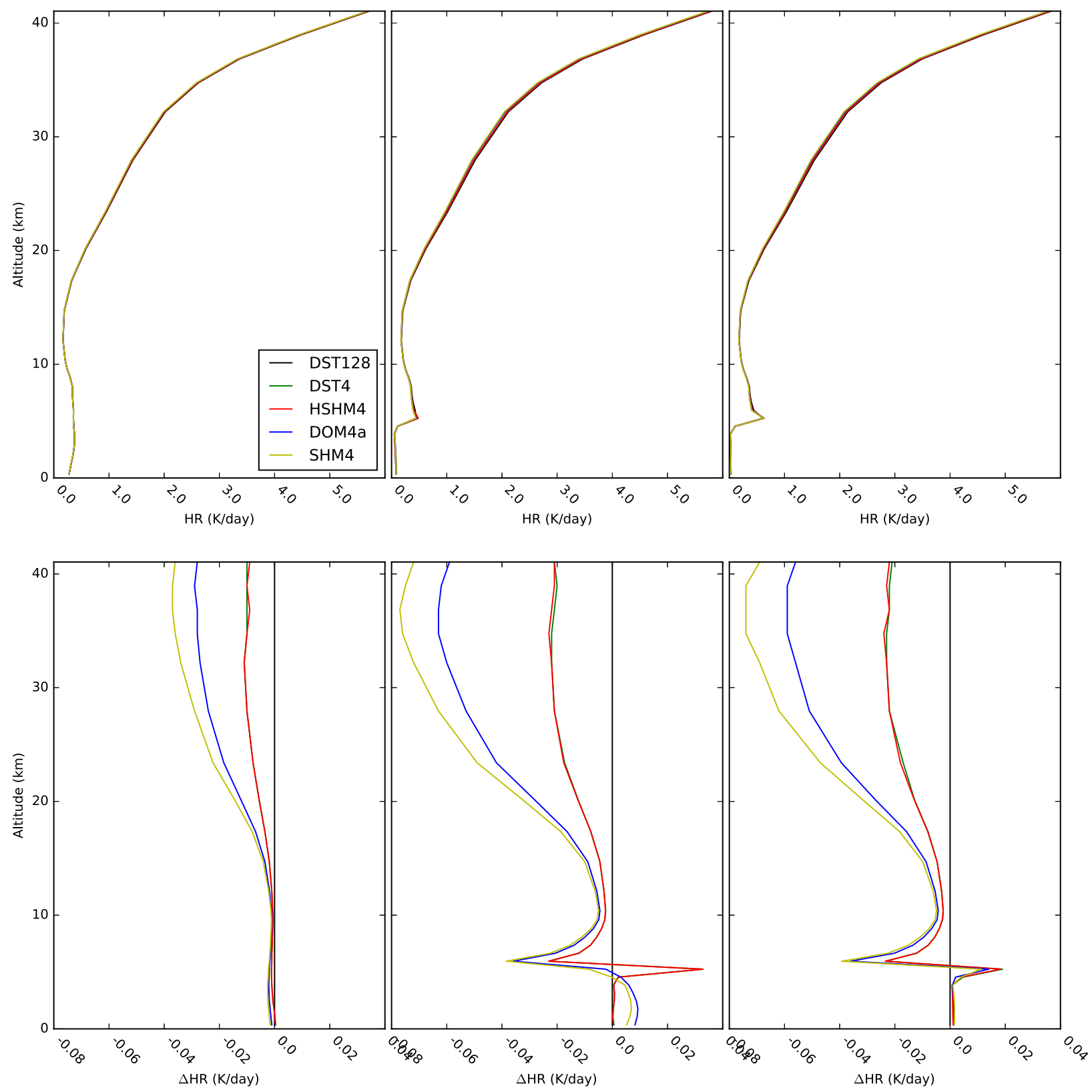

FIG. 8. As in Fig. 7, but for $\mu_{0}=0.1$.

HSHM4 and the DST4 appear a little better than the other two in most cases, but the advantages are not very significant.

Another useful variable for shortwave radiation is the actinic flux, which is essential to the atmospheric photochemical processes and can be defined as

$$
F_{A}=4 \pi\langle I(\mu, \phi)\rangle=4 \pi\langle\mathbf{A}\rangle \cdot \mathbf{I},
$$

where angle brackets have been defined by (I.3). For HSHM4, it is easy to see that

$$
F_{A}=\left[\begin{array}{llll}
2 \pi & \pi & 2 \pi & \pi
\end{array}\right] \cdot \tilde{\mathbf{I}},
$$

where $\tilde{\mathbf{I}}=\mathbf{I}$ for the HSHM4. In practice, the actinic flux is always evaluated for specific spectral intervals that are effective for photochemical reactions; to get an overall picture without involving specific reactions, the actinic fluxes are calculated simply for all shortwave bands under the same nine conditions as in the heating-rate calculations. The results and the relative errors relative to the results of the DST128 are shown in Figs. 10-12. The results of the HSHM4 and the DST4 are significantly better 

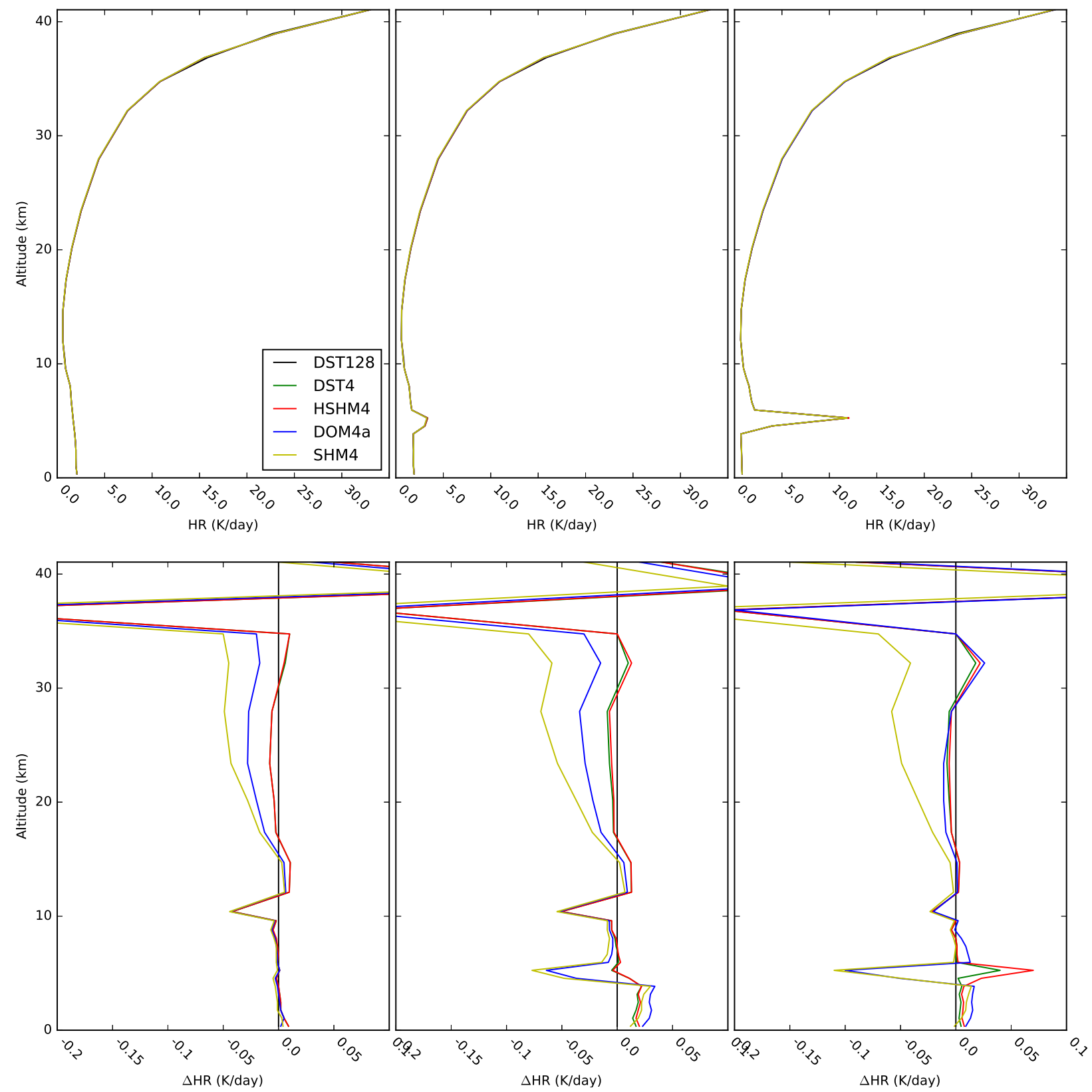

FIG. 9. As in Fig. 7, but for $\mu_{0}=0.9$.

than those of the DOM4a and the SHM4 in most cases. The profiles of the HSHM4 and the DST4 are distinguishable only for the cloudy cases and at the altitudes below the cloud, but the accuracies are similar. For the case of $\mu=0.9$, the profile of the HSHM4 is a little better than that of the DST4 at altitudes above the (thin and thick) cloud layer.

The longwave heating-rate profiles for the U.S. Standard Atmosphere, 1976 (STD), the tropical atmosphere (TRP), and the subarctic winter atmosphere (SAW; McClatchey et al. 1972) are calculated with the same cloud prescriptions mentioned above. The results are shown in Figs. 13-15, along with the errors relative to those of the DST128. It can be seen that the HSHM4 and the DST4 are significantly more accurate than the SHM4 and the DOM4a for all of the three atmosphere profiles and the clear/cloudy conditions, with most of the errors less than $0.1 \mathrm{~K} \mathrm{day}^{-1}$. The HSHM4 always has better results than the DST4 does within the cloud layers. The differences between the HSHM4 and the DST4 above $30 \mathrm{~km}$ are due to the roundoff errors from the different numerical procedures. The DOM4 and the 
TABLE 1. The downward shortwave radiative fluxes at the surface $F_{\mathrm{Srf}}^{-}$, the upward fluxes at the top of the atmosphere $F_{\mathrm{TOA}}^{+}$, and the atmospheric net absorptions $A$, for the $S T D$, calculated with the DST128 for the nine cases of $\mu_{0}$ and LWP and the relative errors $r(\cdot)$ of the corresponding results of the HSHM4, the SHM4, the DOM4a, and the DST4. The boldface type indicates where the accuracy is better than $1 \%$.

\begin{tabular}{|c|c|c|c|c|c|c|c|c|c|c|}
\hline & & \multicolumn{9}{|c|}{$\mu_{0}$} \\
\hline \multirow{2}{*}{\multicolumn{2}{|c|}{$\operatorname{LWP}\left(\mathrm{g} \mathrm{m}^{-2}\right)$}} & \multicolumn{3}{|c|}{0.1} & \multicolumn{3}{|c|}{0.5} & \multicolumn{3}{|c|}{0.9} \\
\hline & & 0 & 10 & 100 & 0 & 10 & 100 & 0 & 10 & 100 \\
\hline \multirow[t]{3}{*}{ DST128 $\left(\mathrm{W} \mathrm{m}^{-2}\right)$} & $F_{\mathrm{Srf}}^{-}$ & 67.57 & 41.42 & 19.22 & 505.2 & 403.7 & 182.4 & 986.0 & 914.5 & 456.6 \\
\hline & $F_{\mathrm{TOA}}^{+}$ & 35.62 & 68.90 & 87.49 & 136.2 & 219.3 & 403.5 & 224.9 & 267.5 & 621.0 \\
\hline & $A^{10 \mathrm{~A}}$ & 46.1 & 33.8 & 32.94 & 138.9 & 136.9 & 129.8 & 208.3 & 222.9 & 235.8 \\
\hline \multirow{3}{*}{ HSHM4 (\%) } & $r\left(F_{\mathrm{Srf}}^{-}\right)$ & +0.06 & -0.9 & +1.2 & +0.04 & -1.3 & -0.5 & +0.05 & +0.2 & +0.2 \\
\hline & $r\left(F_{\mathrm{TOA}}^{+}\right)$ & +0.3 & +0.9 & +0.2 & -0.10 & +1.7 & +0.09 & -0.2 & -0.9 & -0.3 \\
\hline & $r(A)$ & -0.3 & -0.8 & -1.0 & $-\mathbf{0 . 0 3}$ & +0.4 & +0.3 & 0.03 & +0.4 & +0.3 \\
\hline \multirow[t]{3}{*}{ SHM4 (\%) } & $r\left(F_{\mathrm{Srf}}^{-}\right)$ & +0.6 & +5.2 & +2.5 & +0.3 & +1.5 & -0.8 & +0.2 & +0.9 & +0.15 \\
\hline & $r\left(F_{\mathrm{TOA}}^{+}\right)$ & +0.4 & -1.2 & +0.6 & -0.3 & -1.5 & +0.7 & -0.6 & -2.3 & +0.11 \\
\hline & $r(A)$ & -1.0 & -2.6 & -2.8 & -0.5 & -1.2 & -1.2 & -0.3 & -0.3 & -0.5 \\
\hline \multirow[t]{3}{*}{ DOM4a (\%) } & $r\left(F_{\mathrm{Srf}}^{-}\right)$ & +0.4 & +4.2 & +0.8 & +0.09 & +1.0 & -1.5 & +0.07 & +0.5 & -0.3 \\
\hline & $r\left(F_{\mathrm{TOA}}^{+}\right)$ & +0.4 & -1.2 & +0.8 & +0.06 & -1.3 & +0.8 & -0.12 & -1.5 & +0.2 \\
\hline & $r(A)$ & -0.8 & -1.7 & -2.4 & -0.3 & -0.3 & -0.7 & -0.15 & +0.13 & -0.08 \\
\hline \multirow[t]{3}{*}{ DST4 (\%) } & $r\left(F_{\mathrm{Srf}}^{-}\right)$ & +0.06 & -0.6 & +1.7 & +0.04 & -1.0 & +0.3 & +0.05 & +0.002 & -0.15 \\
\hline & $r\left(F_{\mathrm{TOA}}^{+}\right)$ & +0.3 & +0.7 & +0.06 & -0.10 & +1.1 & -0.3 & -0.2 & -0.3 & +0.03 \\
\hline & $r(A)$ & -0.3 & -0.8 & -1.0 & -0.03 & +0.5 & +0.5 & +0.03 & +0.4 & +0.14 \\
\hline
\end{tabular}

SHM4, in contrast, get almost unacceptable large errors in the cloud layers and the lowest layers.

Table 2 lists the upward longwave fluxes at top of the atmosphere, the downward fluxes at the surface, and the net emission of the atmosphere obtained by the DST128 and relative errors of the corresponding results obtained by the other methods for the nine cases mentioned above. The boldface type indicates where the accuracy is better than $1 \%$. The results of the HSHM4 and the DST4 are obviously much better than those of the other two, and the DST4 has better accuracy than the HSHM4 does.

\section{Numerical experiment of modifying HSHM4}

The results for the model atmospheres obtained in the last section show that the four methods all have acceptable accuracies for shortwave radiation, but the HSHM4 and the DST4 (or the DOM4b) are significantly more accurate than the others for longwave radiation. For longwave radiation in the cloud layers, the HSHM4 gets better heating-rate profile but worse flux and general radiation budget than the DST4 does. However, the results of the HSHM4 and the DST4 for longwave heating rate in the cloud layers are significantly less accurate (typically $0.1-0.2 \mathrm{~K} \mathrm{day}^{-1}$ ) than those for shortwave (typically around $0.05 \mathrm{~K} \mathrm{day}^{-1}$ ).

As an example of the facility provided by the general decomposition method for finding improved basis for atmospheric problems, a simple modification to the basis of the HSHM4 is investigated, which introduces a higher degree of polynomials into $H_{1}(\mu)$ [see (3)], that is,

$\mathbf{A}^{\prime}=\left[\begin{array}{llll}H_{0}(\mu) & H_{1}^{\prime}(\mu) & H_{0}(-\mu) & H_{1}^{\prime}(-\mu)\end{array}\right]$,

where

$$
\begin{aligned}
H_{1}^{\prime}(\mu) & =\left\{\begin{array}{ccc}
\mu+\beta P_{2}^{*}(\mu), & \text { when } & \mu \geq 0 \\
0, & \text { when } & \mu<0
\end{array},\right. \\
P_{2}^{*} & =6 \mu^{2}-6 \mu+1,
\end{aligned}
$$

which is a transformation of $P_{2}$ defined in (5) by $\mu \rightarrow 2 \mu-1$, and $\beta$ is the modification factor to be investigated by the numerical experiment.

The modified basis with a parameter provides a family of bases with different $\beta$. The numerical experiment shows that when $\beta=-0.2$, the longwave heating-rate profiles can be generally improved, especially for the thick cloud cases where the errors in the cloud layers are mostly limited within $0.05 \mathrm{~K} \mathrm{day}^{-1}$ (Fig. 16), while the accuracies for the shortwave radiation are little affected; that is, typically maximum errors in the cloud layers are still around $0.05 \mathrm{~K} \mathrm{day}^{-1}$ (not shown). Besides, the longwave fluxes obtained by the modified HSHM4 are listed in Table 3 with the maximum relative errors around $0.3 \%$, which are significantly improved relative to the results obtained by the HSHM4 and the DST4 listed in Table 2. 

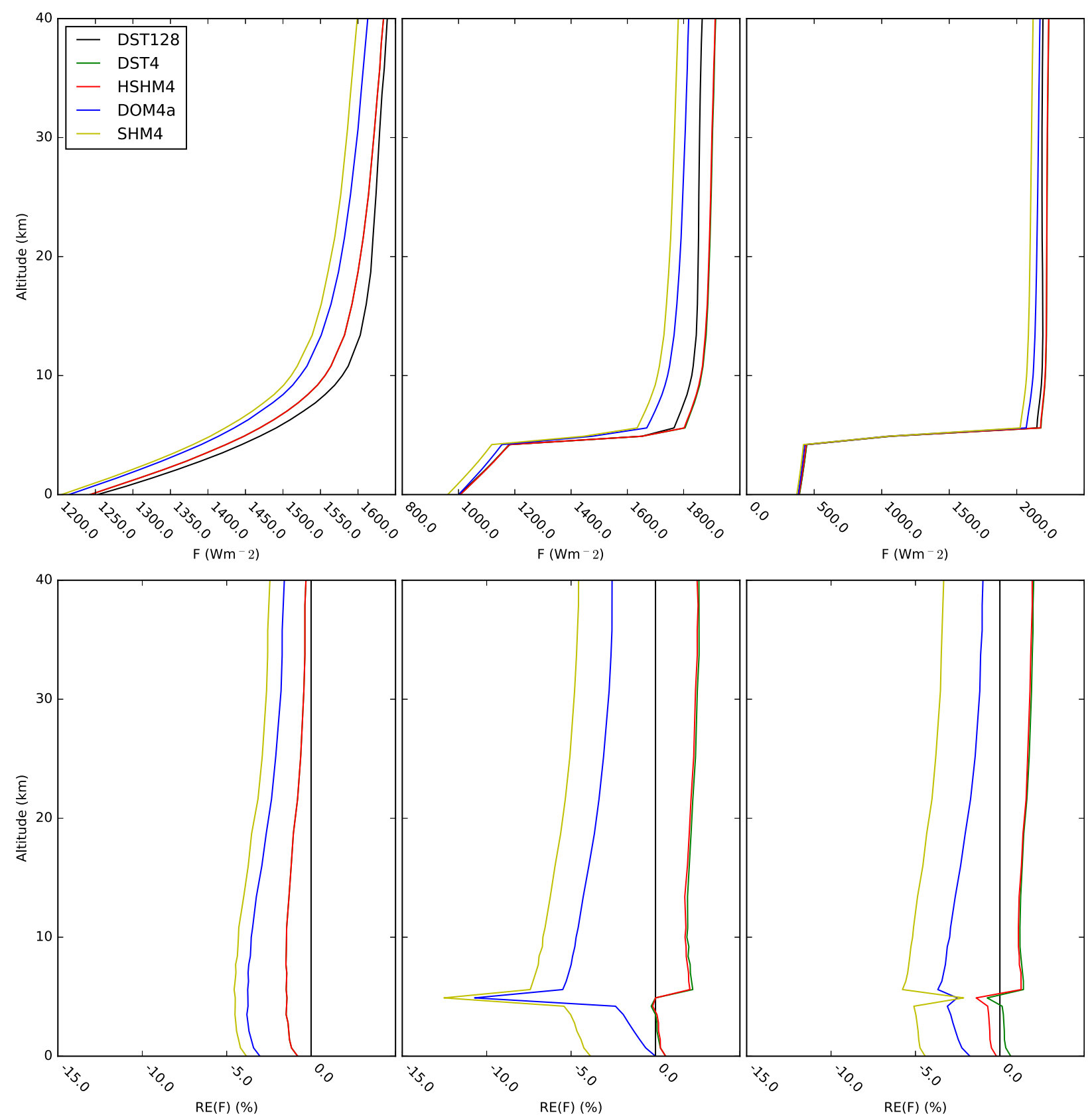

FIG. 10. The profiles of (top) the actinic flux calculated with the DST128, the DST4, the HSHM4, the DOM4a, and the SHM4 for $\mu_{0}=0.5$ and middle-cloud LWP $=$ (left) 0 , (middle) 10 , and (right) $100 \mathrm{~g} \mathrm{~m}^{-2}$ and (bottom) the errors relative to the DST128 results.

\section{Discussion}

The basis for the HSHM4 [(1)] is hemisphere confined and horizon symmetrical. As discussed in section 4a of Part I, the eigenvalue problem of $\mathbf{D}$ can be simplified to that of a $2 \times 2$ matrix, which can be solved analytically. On the other hand, the inversion of the matrices involved in (I.65), (I.44), (I.45), and (I.46) can be obtained analytically in theory so that the analytical solution for homogeneous layers [(I.65)-(I.67)] can be obtained. That will lead to further optimizations of the numerical procedure. Since the purpose of this paper is to illustrate how the unified formulation can be used to develop the prototype of a new radiative transfer method through the general procedure outlined in section 5a of Part I and what the numerical results of the HSHM4 as an example look like, the discussion of further optimization would be off topic as it is indeed basis dependent. A derivation of the analytical solution 

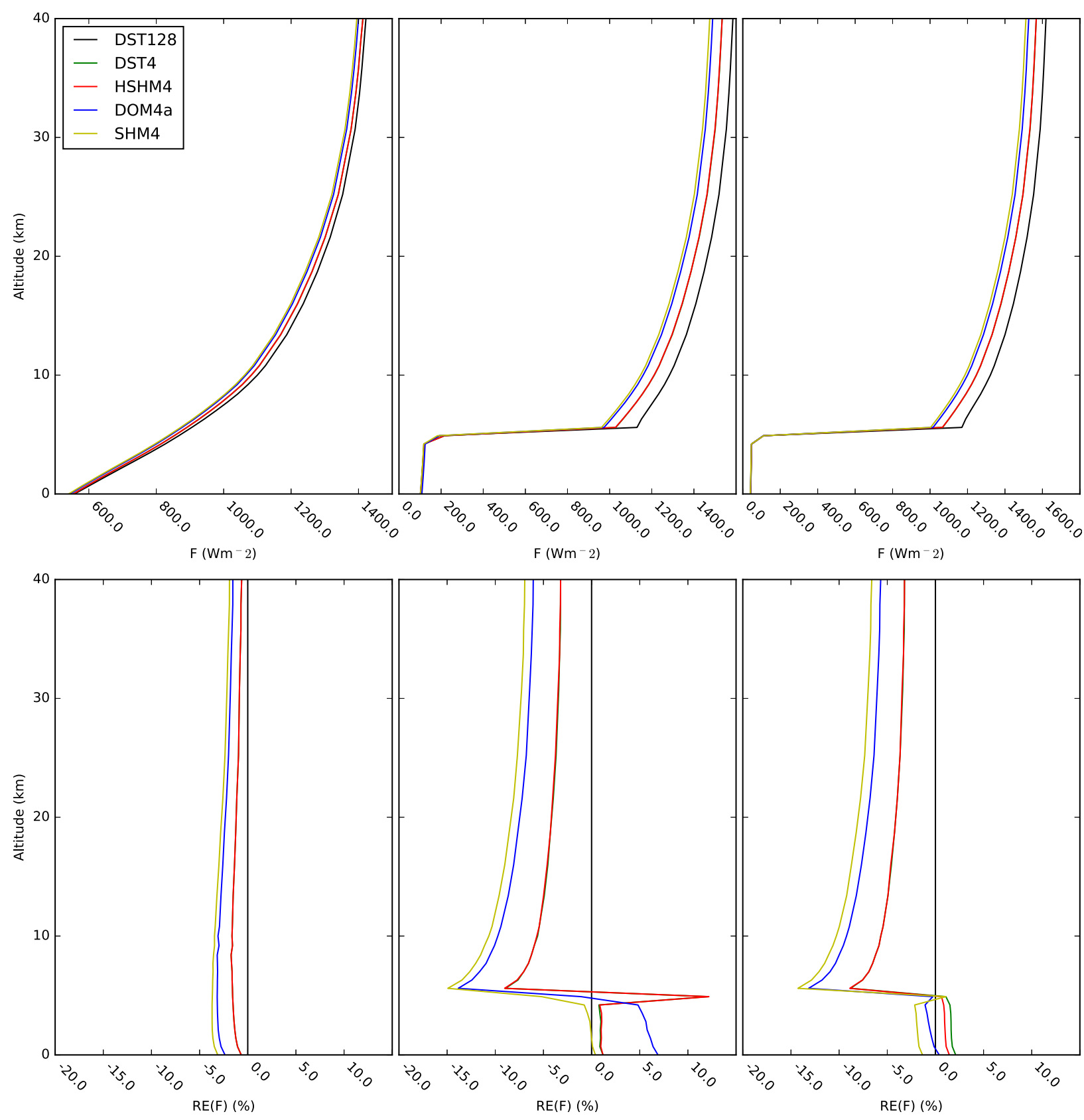

FIG. 11. As in Fig. 10, but for $\mu_{0}=0.1$.

to the eigenvalue problem of $\mathbf{D}$ of the HSHM4 is given in the appendix.

The CPU time is not discussed either for a similar reason. The efficiencies of the methods with the same number of components should be similar in theory, but the specific bases that determine characteristics of the involved matrices will provide more chance of optimization. In their prototypes, the HSHM4, the SHM4, and the DOM4ab take almost the same amount of time because they virtually follow the same numerical procedure.
The large errors of the SHM4 and the DOM4a for the longwave bands and the lower troposphere may be related to their notable errors in the apparent emission for large optical depth (Fig. 6). It is noticeable that the relative errors of the SHM4 and the DOM4a in the surface downward fluxes in Table 2 are consistent to the maximum relative errors in the apparent emissions. The basis functions of the SHM are obviously coupled between the upward and downward hemispheres, while the upward and downward radiation are essentially coupled 

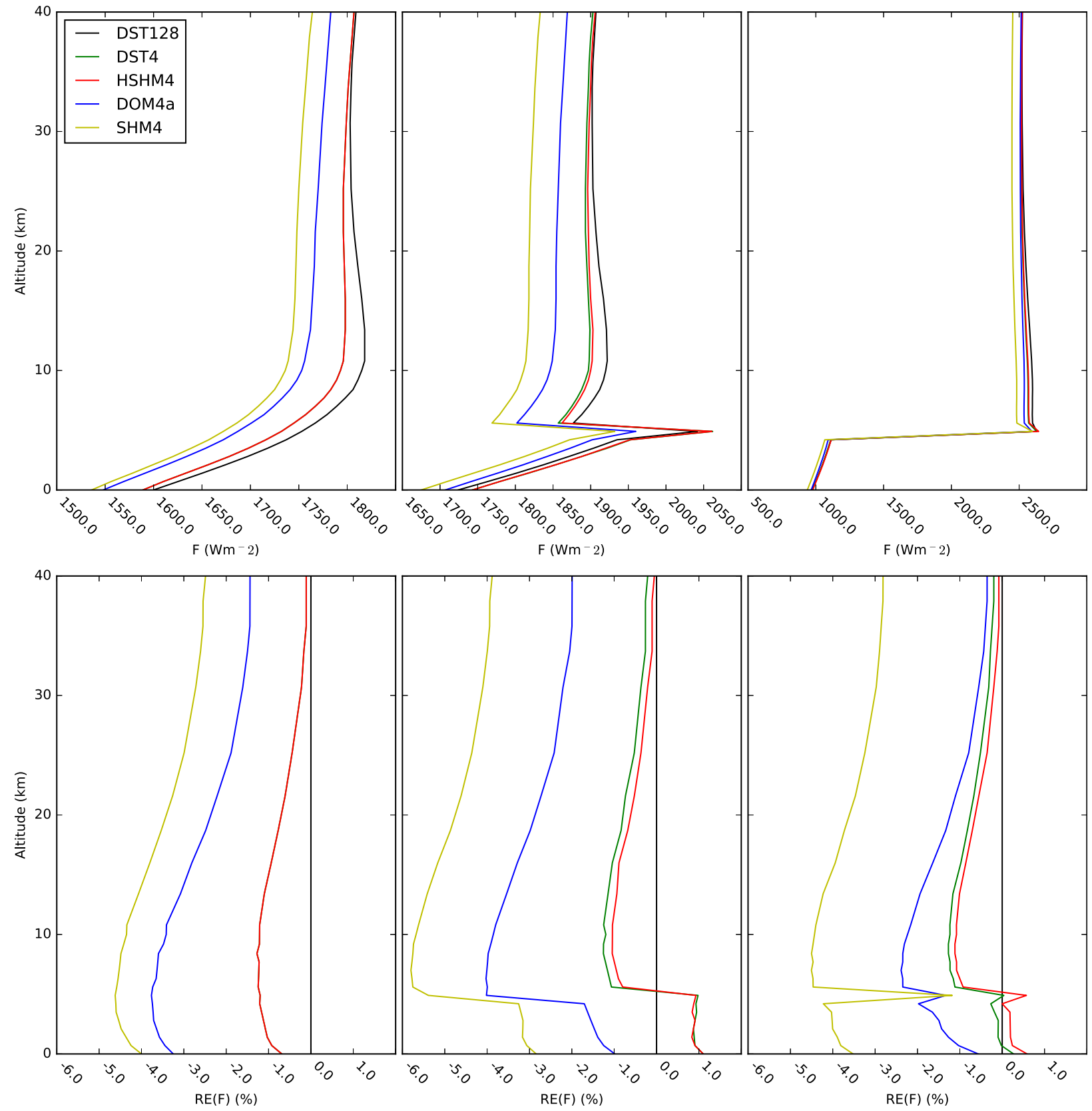

FIG. 12. As in Fig. 10, but for $\mu_{0}=0.9$.

through the scattering processes. Therefore, the errors of the SHM4 in Fig. 6 are less for more scattering media, but the large single-scattering albedos seldom occur in the longwave bands of Earth's atmosphere. This situation for the basis functions of the DOM4a is not so obvious, but they are indeed coupled between the hemispheres as well because the Gaussian quadrature is valid only for the full-range integral, and hence, it cannot handle the discontinuity between the two hemispheres very well.
The HSHM4 can be applied to solve the azimuth angle expansion components of the radiance as well (see section $4 \mathrm{f}$ of Part I) to get the radiance field. That is not discussed however because the low-order approximations are usually used in the calculations of the planar fluxes in atmospheric models where only the azimuth-averaged radiance needs to be explicitly expressed and the angular distribution of the radiance obtained by the low-order methods is generally unreliable. For high-order methods, the completeness of basis (or the family of functions), 

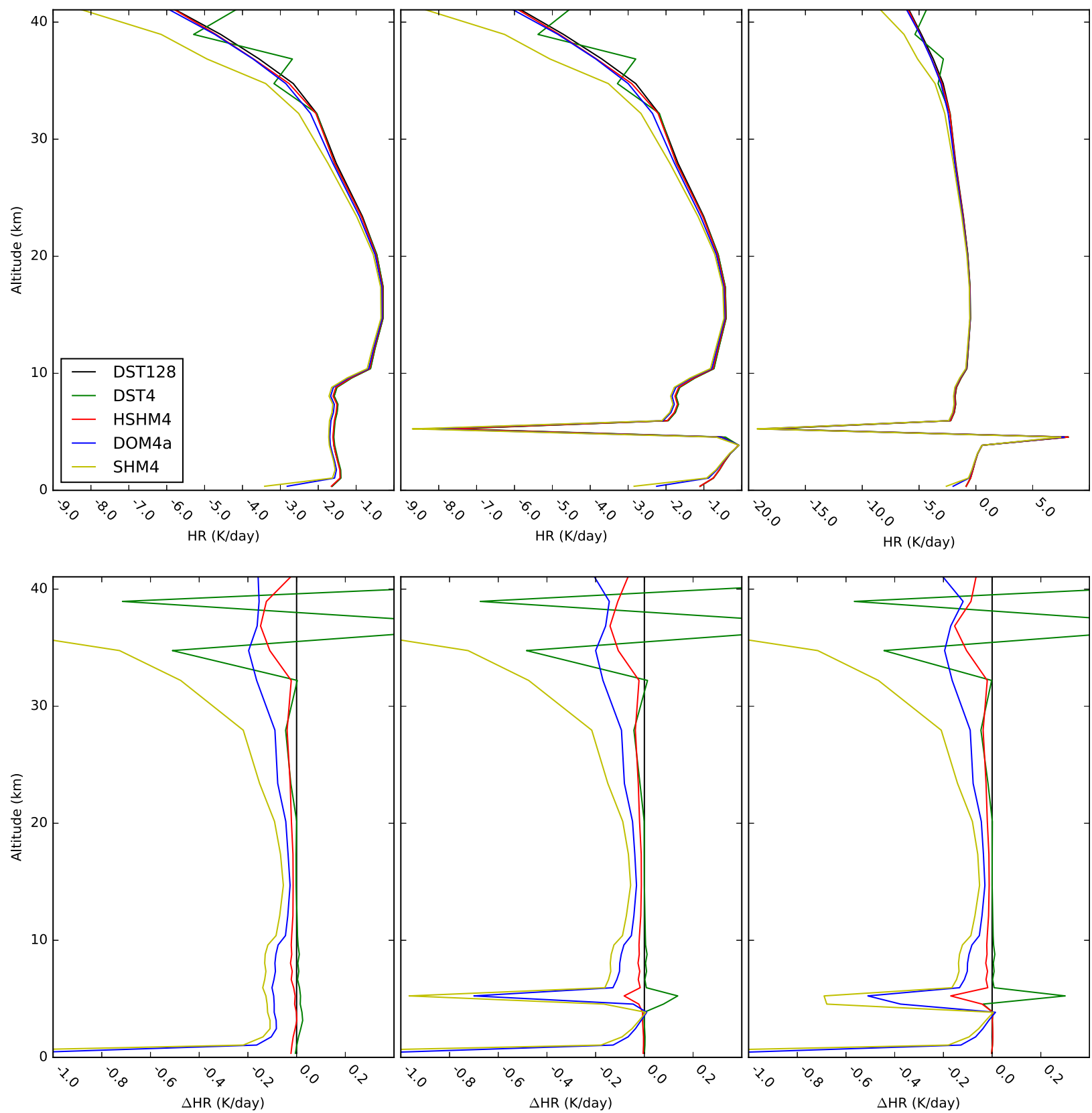

FIG. 13. The profiles of (top) the heating rate calculated with the DST128, the DST4, the HSHM4, the DOM4a, and the SHM4 for the STD and middle-cloud LWP $=$ (left) 0 , (middle) 10 , and (right) $100 \mathrm{~g} \mathrm{~m}^{-2}$ and (bottom) the absolute errors relative to the DST128 results.

which is not required by the low-order method, will be more important. The basis of HSHM4 is not orthogonal in its current state, but it is very easy to get its equivalent and orthogonal alternative by the simple transformation $\mu \rightarrow 2 \mu-1$ that transforms the Legendre polynomials from the full range to the half range, and the completeness of the Legendre polynomials is reserved. Therefore, the HSHM can be considered an exact method like the DOM and the SHM when $N$ approaches infinity.
The similarity between the results of the HSHM4 and the DOM4b is quite noticeable. Thomas and Stamnes (1999, section 7.5.1) showed the two-stream method with the full-range Gaussian quadrature (DOM2a) is equivalent to the Eddington method (SHM2) except for the treatment of boundary conditions, and they also mentioned the two-stream method with the halfrange Gaussian quadrature (DOM2b), which should be logically equivalent to the so-called hemispheric 

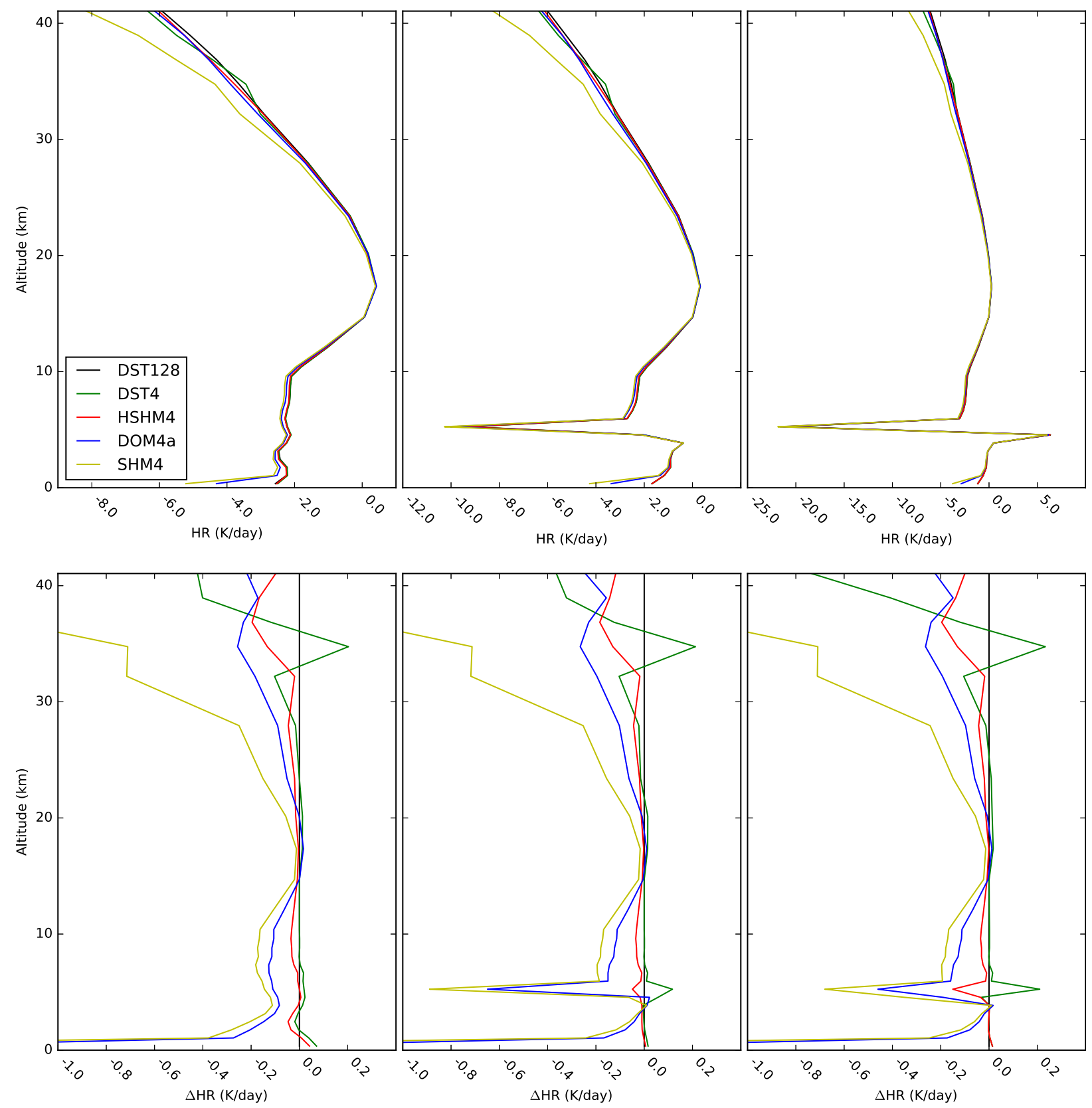

FIG. 14. As in Fig. 13, but for the TRP.

constant method (may be called HSHM2; Meador and Weaver 1980).

With the general decomposition formulation, these similarities can be accounted for analytically, but only a qualitative discussion is presented here. For the GDRTES [see (I.8)], the essential integral for multiple scattering effect is the first fold integral in (I.13), $\langle P(\mu) \cdot \mathbf{A}(\mu)\rangle$, in the case of azimuthal average. For the four-stream methods, as a rule of thumb (Thomas and Stamnes 1999, section 6.8.3), the phase function is expressed as four terms in expansion as in (2), a polynomial of degree 3. For the HSHM4, the integration is analytically solved so that the degree of the integrand does not affect the accuracy. But it is not the case for the DOM4b.

The equivalence between the equations of the DOM4b and the HSHM4 is conditional upon the situation that the integral can be exactly evaluated by the double-Gaussian quadrature. It is well known that the $N$-point Gaussian quadrature or the $2 N$-point 

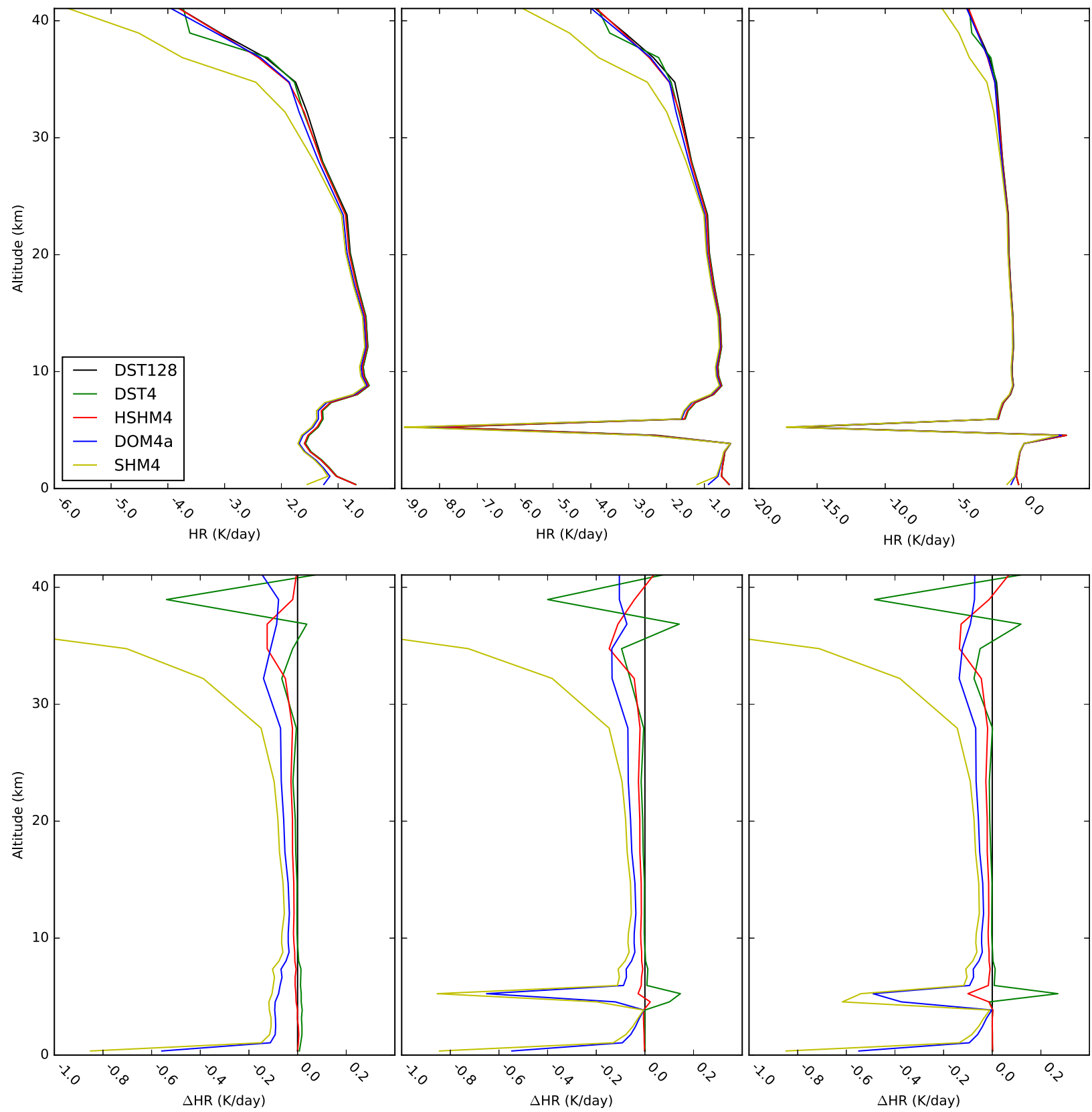

FIG. 15. As in Fig. 13, but for the SAW.

double-Gaussian quadrature gets the exact result for polynomials of degree $2 N-1$, or degree 3 for the four-point double quadrature, for example. In the integration $\langle P(\mu) \cdot \mathbf{A}(\mu)\rangle$, the phase function is a polynomial of degree 3 , and $\mathbf{A}$ represented by two discrete points is equivalent to a linear function, so the integrand is virtually a polynomial of degree 4 and cannot be exactly evaluated by the quadrature rule. Therefore, only when the phase function is expressed as polynomials of degree 2 (with three terms) or less will the results of the HSHM4 and the DOM4b be exactly same, which has been observed in the numerical experiments of this work (not shown).

The discussion above is consistent to the conclusion reached by Thomas and Stamnes (1999) for the twostream case. For the full-range quadrature rule $(N=2)$, the maximum degree of the integrand can be 3 for the exact integration, and $\mathbf{A}$ has degree 1, so the phase function can be expressed with polynomials of degree 2 (one more than the rule of thumb), while the accuracy of 
TABLE 2. The downward longwave radiative fluxes at the surface $F_{\text {Srf }}^{-}$, the upward fluxes at the top of the atmospheres $F_{\text {TOA }}^{+}$, and the atmospheric net emissions $E$, calculated with the DST128 for the nine cases of atmospheric model and LWP and the relative errors $r(\cdot)$ of the corresponding results of the HSHM4, the SHM4, the DOM4a, and the DST4. The boldface type indicates where the accuracy is better than $1 \%$.

\begin{tabular}{|c|c|c|c|c|c|c|c|c|c|c|}
\hline & & \multicolumn{9}{|c|}{ Model } \\
\hline & & \multicolumn{3}{|c|}{ TRP } & \multicolumn{3}{|c|}{ SAW } & \multicolumn{3}{|c|}{$S T D$} \\
\hline \multicolumn{2}{|c|}{$\operatorname{LWP}\left(\mathrm{g} \mathrm{m}^{-2}\right)$} & 0 & 10 & 100 & 0 & 10 & 100 & 0 & 10 & 100 \\
\hline \multirow[t]{3}{*}{ DST128 $\left(\mathrm{W} \mathrm{m}^{2}\right)$} & $F_{\mathrm{Srf}}^{-}$ & 398.7 & 415.3 & 426.6 & 117.8 & 213.2 & 231.8 & 294.1 & 325.0 & 342.9 \\
\hline & $F_{\mathrm{TOA}}^{+}$ & 277.3 & 247.1 & 227.9 & 195.7 & 173.5 & 160.3 & 251.9 & 214.8 & 193.5 \\
\hline & $E$ & 222.4 & 209.0 & 200.9 & 126.0 & 139.2 & 144.6 & 157.3 & 151.1 & 147.7 \\
\hline \multirow[t]{3}{*}{ HSHM4 (\%) } & $r\left(F_{\mathrm{Srf}}^{-}\right)$ & -0.06 & +0.07 & +0.03 & -0.3 & -0.16 & +0.03 & -0.06 & -0.07 & +0.08 \\
\hline & $r\left(F_{\mathrm{TOA}}^{+}\right)$ & +0.7 & +0.9 & +0.9 & +0.7 & +0.9 & +0.9 & +0.8 & +1.1 & +1.2 \\
\hline & $r(E)$ & +0.8 & +1.0 & +1.1 & +0.7 & +0.9 & +1.1 & +1.2 & +1.4 & +1.7 \\
\hline \multirow[t]{3}{*}{ SHM4 (\%) } & $r\left(F_{\mathrm{Srf}}^{-}\right)$ & +7.6 & +7.2 & +6.9 & +7.8 & +7.5 & +6.8 & +7.5 & +7.5 & +7.0 \\
\hline & $r\left(F_{\mathrm{TOA}}^{+}\right)$ & +2.9 & +3.0 & +3.7 & +2.6 & +3.2 & +4.3 & +2.2 & +2.7 & +3.9 \\
\hline & $r(E)$ & +17 & +18 & +19 & +15 & +16 & +16 & +18 & +20 & +21 \\
\hline \multirow[t]{3}{*}{ DOM4a (\%) } & $r\left(F_{\mathrm{Srf}}^{-}\right)$ & +5.0 & +4.7 & +4.5 & +4.9 & +4.8 & +4.4 & +4.8 & +4.8 & +4.5 \\
\hline & $r\left(F_{\mathrm{TOA}}^{+}\right)$ & +3.0 & +3.1 & +3.6 & +2.7 & +3.1 & +3.9 & +2.7 & +3.1 & +4.0 \\
\hline & $r(E)$ & +13 & +13 & +14 & +11 & +11 & +11 & +13 & +15 & +16 \\
\hline \multirow[t]{3}{*}{ DST4 (\%) } & $r\left(F_{\mathrm{Srf}}^{-}\right)$ & +0.11 & -0.003 & +0.03 & +0.2 & -0.008 & +0.03 & +0.4 & 0.08 & +0.08 \\
\hline & $r\left(F_{\mathrm{TOA}}^{+}\right)$ & -0.7 & -0.7 & -0.8 & -0.4 & -0.3 & -0.4 & -0.6 & -0.6 & -0.7 \\
\hline & $r(A)$ & -0.7 & -0.8 & -0.8 & -0.3 & -0.4 & -0.4 & -0.2 & -0.6 & -0.7 \\
\hline
\end{tabular}

the integral can be kept. For the case of half-range quadrature $(N=1)$, the maximum degree of the integrand for the exact integration is 1 , and $\mathbf{A}$ has degree 0 (with only one point in each half range), so the phase function can be expressed as a linear function, a polynomial of degree 1 , just as the rule of thumb indicates.

As a summary of the discussion above, the equivalence between the radiative transfer equations of the DOMs with the Gaussian or the double-Gaussian quadrature rule and those of the SHMs or the HSHMs, respectively, is valid only when the phase function is expressed as polynomials of $\mu$ and within a limited degree. When the phase function is expressed as polynomials of degree 3 , as usual for the fourcomponent methods, the HSHM4 and the DOM4b yield different results and they are generally different methods.

As discussed in Part I, the advantage of a specific basis depends on the atmospheric model concerned. Although the HSHM4 and the DST4 (or the DOM4b) behave better in most cases for Earth's atmosphere, it can be seen in some cases or at some altitudes of the atmosphere that the other methods get better heatingrate or flux results. That implies there is no general criterion about the bases or there does not exist the best basis a priori for all cases. However, further investigation about the characteristics of the radiance angular distribution in the atmospheres will provide useful guidance for finding better basis atmospheric problems.

\section{Conclusions}

After introducing the unified formulation for radiative transfer based on the general decomposition of radiance and the general procedure to develop new methods in Part I, an example of the HSHM4 is given in this work to show the simplicity and feasibility of the general formulation and method as a metaalgorithm. The prototypes of the established methods such as the two-stream discrete ordinate method (DOM2), the Eddington method (SHM2), the fourstream discrete ordinate methods (DOM4ab), and the spherical harmonic method with four components (SHM4) can be reproduced easily from the general procedure. While detailed discussions about the established methods are not necessary, some of the numerical results of the DOM4ab and SHM4 have been used in comparison with those of the HSHM4. The same results yielded by the DOM4b and the DST4 can be considered a validation of the general formulation.

The planar flux reflection, transmission, and absorption of homogeneous layer calculated with the HSHM4, the DOM4b, the DOM4a, and SHM4 have acceptable accuracy for most cases. The apparent emissions obtained by the HSHM4 and the DOM4b converge to the theoretical value when there is not scattering effect, while those of the DOM4a and the SHM4 fail to. The results of the shortwave heating rate and the actinic flux of the HSHM4 and the DOM4b are slightly better than 

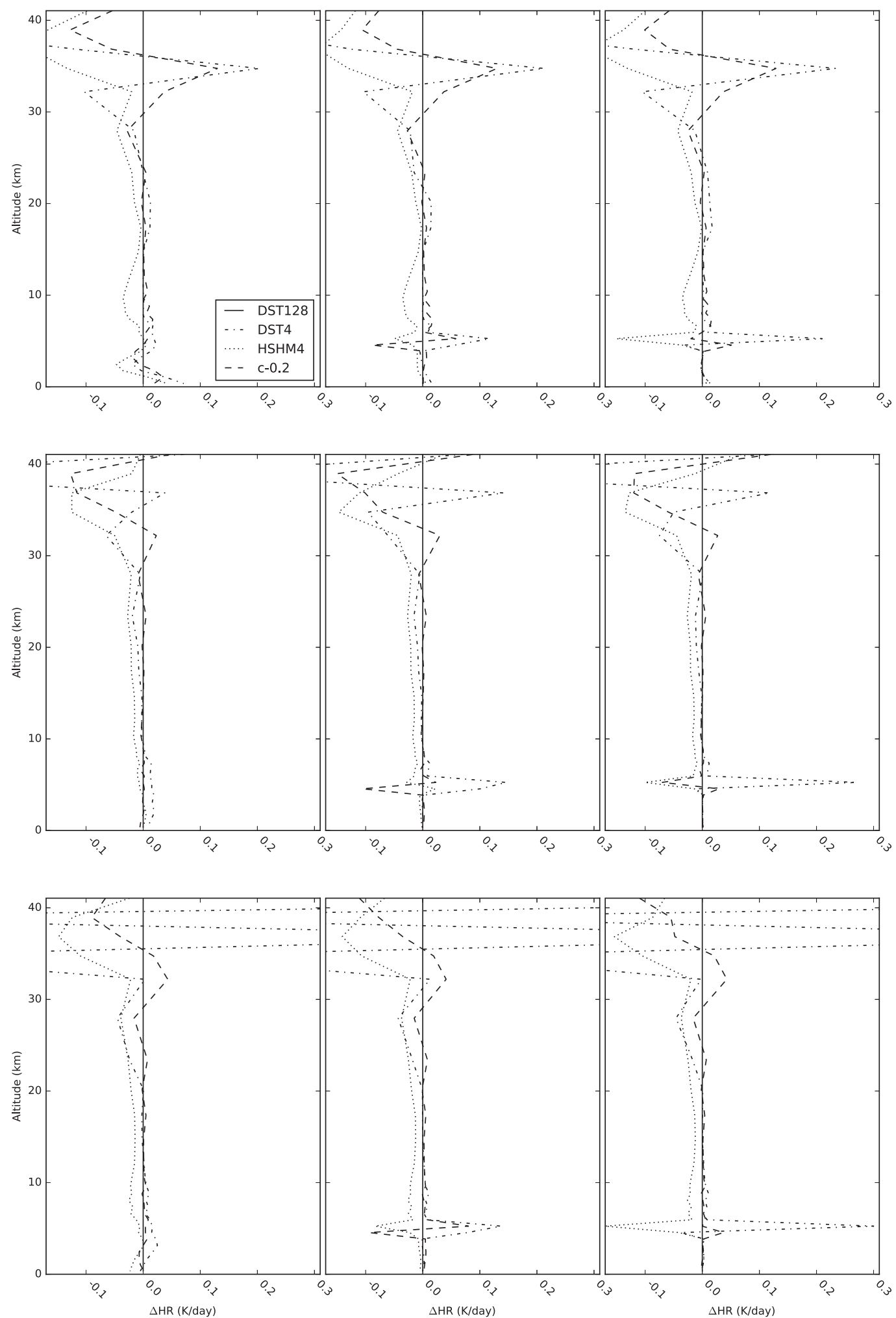

FIG. 16. The profiles of the errors of the cooling rate calculated by the DST4, the HSHM4, and the modified HSHM4 (c-0.2) relative to the results of the DST128 for (top) the TRP, (middle) the SAW, and (bottom) the STD and for the middle-cloud LWP $=$ (left) 0 , (middle) 10 , and (right) $100 \mathrm{~g} \mathrm{~m}^{-2}$. 
TABLE 3. The relative errors of the downward longwave radiative fluxes at the surface $F_{\mathrm{Srf}}^{-}$, the upward fluxes at the top of the atmospheres $F_{\mathrm{TOA}}^{+}$, and the atmospheric net emissions $E$, calculated by the modified HSHM4 relative $r(\cdot)$ to the results of the DST128 for the nine cases of atmospheric model and LWP.

\begin{tabular}{|c|c|c|c|c|c|c|c|c|c|c|}
\hline & & \multicolumn{9}{|c|}{ Model } \\
\hline \multirow{2}{*}{\multicolumn{2}{|c|}{$\operatorname{LWP}\left(\mathrm{g} \mathrm{m}^{-2}\right)$}} & \multicolumn{3}{|c|}{ TRP } & \multicolumn{3}{|c|}{ SAW } & \multicolumn{3}{|c|}{$S T D$} \\
\hline & & 0 & 10 & 100 & 0 & 10 & 100 & 0 & 10 & 100 \\
\hline \multirow[t]{3}{*}{ Modified HSHM4 (\%) } & $r\left(F_{\mathrm{Srf}}^{-}\right)$ & 0.005 & 0.14 & 0.013 & -0.018 & 0.15 & 0.03 & 0.2 & 0.15 & 0.05 \\
\hline & $r\left(F_{\mathrm{TOA}}^{+}\right)$ & -0.3 & -0.3 & -0.3 & 0.07 & 0.07 & 0.10 & -0.2 & -0.3 & -0.2 \\
\hline & $r(E)$ & -0.3 & -0.14 & -0.3 & 0.09 & 0.3 & 0.15 & -0.015 & -0.14 & -0.2 \\
\hline
\end{tabular}

those of the others in most cases, especially for the case of small cosine of solar zenith angle and for the cloud layers. The results of longwave cooling rate and flux of the HSHM4 and the DOM4b are much improved relative to those of the others. In terms of cooling rate of the longwave radiation, the HSHM4 is more accurate than the DOM4b, with the errors reduced by more than a half, but in terms of general energy budget of the system, the DOM4b is slightly more accurate than the HSHM4. The primary numerical experiment shows the possibility that the HSHM4 can be further modified to be more accurate for the longwave problems.

The HSHM4, like the DOM4b, can solve the problems involving both the multiple scattering and the thermal emission, and that makes it useful for the modeler interested in more careful treatment of the longwave radiative transfer. It is a common practice in the current climate models to switch between different radiative transfer methods for shortwave and longwave bands and sometimes to calculate twice with both for the bands around $4 \mu \mathrm{m}$. The HSHM 4 can be deployed consistently to all of the bands without artificial division between the longwave and shortwave, and the sources of solar beam and the thermal emission within the terrestrial-atmospheric system can be treated together. This advantage and its good accuracies in the heating rate, the planar flux, and the actinic flux make the HSHM4 a promising method for climate modeling studies, though further refinement and optimization are needed.

The HSHM4 and the numerical results are presented here not only to introduce a new scheme of radiative transfer for atmospheric sciences but more as an example of the applications of the unified formulation established in Part I, which relates the prescribed basis directly to the prototype model and has been shown as a handy tool to explore new radiative transfer methods.

Acknowledgments. This work is sponsored by Chinese NSFC projects (Grants 41590875 and 41075077).

\section{APPENDIX}

\section{An Analytical Solution to the Eigenvalue Problem in the HSHM4}

The eigenvalue problem of the matrix with the same blocking structure as $\mathbf{D}$ [see (8)] has been discussed by Stamnes and Swanson (1981). If an eigenvector of $\mathbf{D}$ is written as

$$
\mathbf{g}=\left[\begin{array}{l}
\mathbf{g}^{+} \\
\mathbf{g}^{-}
\end{array}\right]
$$

and the eigenvalue is written as $k$, the eigenvalue problem

$$
\mathbf{D} \cdot \mathbf{g}=k \mathbf{g}
$$

leads to

$$
\left(\mathbf{D}_{f}+\mathbf{D}_{b}\right) \cdot\left(\mathbf{g}^{+}+\mathbf{g}^{-}\right)=k\left(\mathbf{g}^{+}-\mathbf{g}^{-}\right)
$$

and

$$
\mathbf{D}^{\prime} \cdot\left(\mathbf{g}^{+}+\mathbf{g}^{-}\right)=k^{2}\left(\mathbf{g}^{+}+\mathbf{g}^{-}\right)
$$

where

$$
\mathbf{D}^{\prime}=\left[d_{i j}^{\prime}\right]_{2 \times 2}=\left(\mathbf{D}_{f}-\mathbf{D}_{b}\right) \cdot\left(\mathbf{D}_{f}+\mathbf{D}_{b}\right),
$$

and

$d_{11}^{\prime}=\frac{3}{16}(\varpi-1)\left(16 g_{1} \varpi+63 g_{3} \varpi-160\right)$,

$d_{12}^{\prime}=\frac{1}{256}\left(\begin{array}{c}384 g_{1} \varpi^{2}-384 g_{1} \varpi-945 g_{2} g_{3} \varpi^{2} \\ +2160 g_{2} \varpi+1512 g_{3} \varpi^{2}-504 g_{3} \varpi \\ -3840 \varpi+1536\end{array}\right)$,

$d_{21}^{\prime}=-\frac{9}{4}(\varpi-1)\left(7 g_{3} \varpi-16\right)$,

$d_{22}^{\prime}=\frac{3}{64}\left(7 g_{3} \varpi-16\right)\left(15 g_{2} \varpi-24 \varpi+8\right)$. 
The eigenvalue $k^{2}$ of $\mathbf{D}^{\prime}$ can be solved analytically; the results are

$$
\begin{aligned}
& k_{1}^{2}=\alpha-\sqrt{\beta^{2}+\gamma}, \\
& k_{2}^{2}=\alpha+\sqrt{\beta^{2}+\gamma},
\end{aligned}
$$

and the eigenvectors are, respectively,

$$
\begin{aligned}
& \mathbf{g}_{1}^{\prime}=\left[\begin{array}{c}
-\frac{d_{12}^{\prime}}{\beta+\sqrt{\beta^{2}+\gamma}} \\
1
\end{array}\right], \\
& \mathbf{g}_{2}^{\prime}=\left[\begin{array}{c}
-\frac{d_{12}^{\prime}}{\beta-\sqrt{\beta^{2}+\gamma}} \\
1
\end{array}\right],
\end{aligned}
$$

where

$$
\begin{aligned}
& \alpha=\frac{1}{2}\left(d_{11}^{\prime}+d_{22}^{\prime}\right), \\
& \beta=\frac{1}{2}\left(d_{11}^{\prime}-d_{22}^{\prime}\right), \\
& \gamma=d_{12}^{\prime} d_{21}^{\prime} .
\end{aligned}
$$

With the aid of (A3), $\mathbf{k}$ and $\mathbf{G}$ in (I.41) can be written as

$$
\mathbf{k}=\operatorname{diag}\left(k_{1},-k_{1}, k_{2},-k_{2},\right)
$$

and

$$
\begin{aligned}
\mathbf{G} & =\left[\begin{array}{llll}
\mathbf{g}_{1} & \mathbf{g}_{2} & \mathbf{g}_{3} & \mathbf{g}_{4}
\end{array}\right] \\
& =\left[\begin{array}{llll}
\mathbf{G}_{1}^{+} & \mathbf{G}_{1}^{-} & \mathbf{G}_{2}^{+} & \mathbf{G}_{2}^{-} \\
\mathbf{G}_{1}^{-} & \mathbf{G}_{1}^{+} & \mathbf{G}_{2}^{-} & \mathbf{G}_{2}^{+}
\end{array}\right],
\end{aligned}
$$

where

$$
\mathbf{G}_{i}^{ \pm}=\frac{1}{2}\left[1_{2} \pm\left(\mathbf{D}_{f}+\mathbf{D}_{b}\right) / k_{i}\right] \cdot \mathbf{g}_{i}^{\prime} .
$$

\section{REFERENCES}

COESA, 1976: U.S. Standard Atmosphere, 1976. NOAA, 227 pp.

$\mathrm{Hu}$, Y. X., and K. Stamnes, 1993: An accurate parameterization of the radiative properties of water clouds suitable for use in climate models. J. Climate, 6, 728-742, doi:10.1175/ 1520-0442(1993)006<0728:AAPOTR > 2.0.CO;2.

King, M. D., and Harshvardhan, 1986: Comparative accuracy of selected multiple scattering approximations. J. Atmos. Sci., 43, 784 801, doi:10.1175/1520-0469(1986)043<0784:CAOSMS > 2.0.CO;2.

Li, J., and V. Ramaswamy, 1996: Four-stream spherical harmonic expansion approximation for solar radiative transfer. J. Atmos. Sci., 53, 1174 1186, doi:10.1175/1520-0469(1996)053<1174:FSSHEA > 2.0.CO;2

Liou, K.-N., Q. Fu, and T. P. Ackerman, 1988: A simple formulation of the delta-four-stream approximation for radiative transfer parameterizations. J. Atmos. Sci., 45, 1940-1947, doi:10.1175/1520-0469(1988)045<1940:ASFOTD>2.0.CO;2.

McClatchey, R. A., R. E. Fenn, J. E. A. Salby, F. E. Volz, and J. S. Garing, 1972: Optical properties of the atmosphere. Air Force Cambridge Research Laboratories Tech. Rep. AFCRL-71-0279, 85 pp.

Meador, W. E., and W. R. Weaver, 1980: Two-stream approximations to radiative transfer in planetary atmospheres: A unified description of existing methods and a new improvement. J. Atmos. Sci., 37, 630643, doi:10.1175/1520-0469(1980)037<0630:TSATRT >2.0.CO;2.

Stamnes, K., and R. A. Swanson, 1981: A new look at the discrete ordinate method for radiative transfer calculations in anisotropically scattering atmospheres. J. Atmos. Sci., 38, 387-399, doi:10.1175/1520-0469(1981)038<0387:ANLATD>2.0.CO;2. , S.-C. Tsay, W. Wiscombe, and K. Jayaweera, 1988: Numerically stable algorithm for discrete-ordinate-method radiative transfer in multiple scattering and emitting layered media. Appl. Opt., 27, 2502-2509, doi:10.1364/AO.27.002502.

Thomas, G. E., and K. Stamnes, 1999: Radiative Transfer in the Atmosphere and Ocean. Cambridge University Press, 540 pp.

Wang, B., 2017: A unified formulation of radiative transfer in planeparallel atmospheres based on general decomposition of radiance. Part I: The theory. J. Atmos. Sci., https://doi.org/10.1175/ JAS-D-16-0272.1, 4139-4151.

- , H. Liu, and G. Shi, 2000: Radiation and cloud schemes. IAP Global Ocean-Atmosphere-Land System Model, X. Zhang et al., Eds., Science Press, 28-49.

Wiscombe, W. J., 1977: The delta- $M$ method: Rapid yet accurate radiative flux calculations for strongly asymmetric phase functions. J. Atmos. Sci., 34, 1408-1422, doi:10.1175/1520-0469(1977)034<1408 TDMRYA $>2.0 . \mathrm{CO} ; 2$.

Zhang, F., and J. Li, 2013: Doubling-adding method for delta-fourstream spherical harmonic expansion approximation in radiative transfer parameterization. J. Atmos. Sci., 70, 3084-3101, doi:10.1175/JAS-D-12-0334.1. 